William R. Wiley

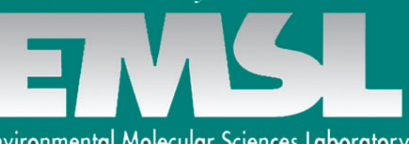

Environmental Molecular Sciences Laboratory

\title{
A Computational Approach to Understanding Oxidant Chemistry and Aerosol Formation in the Troposphere
}

$\begin{array}{ll}\text { B.C. Garrett } & \text { S.M. Kathmann } \\ \text { R. Bianco } & \text { T.J. Lee } \\ \text { L.X. Dang } & \text { A. Morita } \\ \text { D.A. Dixon } & \text { K.A. Peterson } \\ \text { M. Dupuis } & \text { G.K. Schenter } \\ \text { J.S. Francisco } & \text { J.H. Seinfeld } \\ \text { B. Gertner } & \text { S.S. Xantheas } \\ \text { J.T. Hynes } & \end{array}$

August 2002

Theory, Modeling

and Simulation

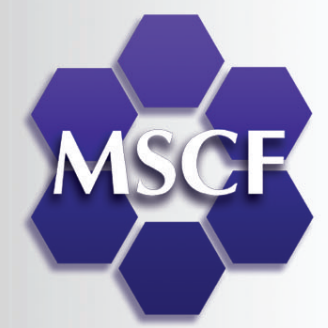


This research was performed in part using the Molecular Science Computing Facility (MSCF) in the William R. Wiley Environmental Molecular Sciences Laboratory, a national scientific user facility sponsored by the U.S. Department of Energy's Office of Biological and Environmental Research and located at the Pacific Northwest National Laboratory. Pacific Northwest is operated for the Department of Energy by Battelle.

\title{
DISCLAIMER
}

This report was prepared as an account of work sponsored by an agency of the United States Government. Neither the United States Government nor any agency thereof, nor Battelle Memorial Institute, nor any of their employees, makes any warranty, express or implied, or assumes any legal liability or responsibility for the accuracy, completeness, or usefulness of any information, apparatus, product, or process disclosed, or represents that its use would not infringe privately owned rights. Reference herein to any specific commercial product, process, or service by trade name, trademark, manufacturer, or otherwise does not necessarily constitute or imply its endorsement, recommendation, or favoring by the United States Government or any agency thereof, or Battelle Memorial Institute. The views and opinions of authors expressed herein do not necessarily state or reflect those of the United States Government or any agency thereof.

\author{
PACIFIC NORTHWEST NATIONAL LABORATORY \\ operated by \\ BATTELLE \\ for the \\ UNITED STATES DEPARTMENT OF ENERGY \\ under Contract DE-ACO6-76RLO183O
}

http://www.pnl.gov/main/publications/external/technical_reports/pnnl-14009.pdf

PNNL-14009, Published by Pacific Northwest National Laboratory for the

Environmental Molecular Sciences Laboratory 


\section{A Computational Approach to Understanding Oxidant \\ Chemistry and Aerosol Formation in the Troposphere}

$\begin{array}{ll}\text { B.C. Garrett } & \text { S.M. Kathmann } \\ \text { R. Bianco } & \text { T.J. Lee } \\ \text { L.X. Dang } & \text { A. Morita } \\ \text { D.A. Dixon } & \text { K.A. Peterson } \\ \text { M. Dupuis } & \text { G.K. Schenter } \\ \text { J.S. Francisco } & \text { J.H. Seinfeld } \\ \text { B. Gertner } & \text { S.S. Xantheas } \\ \text { J.T. Hynes } & \end{array}$

August 2002

Published by Pacific Northwest National Laboratory for the Environmental Molecular Sciences Laboratory 


\title{
A Computational Approach to Understanding Oxidant Chemistry and Aerosol Formation in the Troposphere
}

\begin{abstract}
$\underline{\text { Abstract }}$
Ozone production and aerosol formation in the troposphere are recognized as two major effects of energy-related air pollutants. Tropospheric ozone is of concern primarily because of its impact on health. Ozone levels are controlled by $\mathrm{NO}_{\mathrm{x}}$ and by volatile organic compounds (VOCs) in the lower troposphere. The VOCs can either be from natural emissions from such sources as vegetation and phytoplankton or from anthropogenic sources such as automobiles and oil-fueled power production plants. It is of critical importance to the Department of Energy (DOE) in developing national energy use policies to understand the role of VOCs in determining air quality and how VOC emission or $\mathrm{NO}_{\mathrm{x}}$ emission control strategies should be designed. Atmospheric aerosols are of concern because of their affect visibility, climate, and human health. Equally important, aerosols can change the chemistry of the atmosphere, in dramatic fashion, by providing new chemical pathways (in the condensed phase) that are not available in the gas phase. The oxidation of VOCs and organic sulfur compounds can form precursor molecules that nucleate aerosols. DOE's Atmospheric Chemistry Program has identified the need to evaluate the causes of variations in tropospheric aerosol chemical composition and concentrations, including determining the sources of aerosol particles and the fraction that are of primary and secondary origin. A fundamental understanding of mechanisms for production of oxidants and aerosols in the troposphere is currently not available. We propose the use of advanced theoretical techniques to model the molecular processes that control ozone and aerosol formation.
\end{abstract}

Accurate electronic structure methods will be used to unravel the chemical reactivity of the important naturally occurring VOCs, isoprene, and the organosulfur compound, dimethylsulfide (DMS), that is important in marine environments. Thermochemical data for isoprene, DMS, and their degradation products, detailed mechanistic information about the degradation pathways, and reaction rate constants for key elementary reactions and will be obtained. Molecular simulations will be used to understand the factors controlling aerosol formation by nucleation in binary and ternary systems of sulfuric acid, water, and ammonia. High level ab initio electronic structure calculations will be used to calculate interaction energies and reaction energetics, including reaction profiles, that are crucial to obtaining accurate potential energy functions, the potential energy functions will be used in molecular simulations of the kinetics of cluster formation and degradation, and the kinetic data will be used to model the process of nucleation.

\section{$\underline{\text { Research Team Members }}$}

Bruce C. Garrett, Michel Dupuis, David A. Dixon, Liem X. Dang, Shawn M. Kathmann, Gregory K. Schenter, and Sotiris S. Xantheas

Environmental Molecular Sciences Laboratory, Pacific Northwest National Laboratory, Richland, Washington 99352 
Joseph S. Francisco

Department of Chemistry, Purdue University, West Lafayette, Indiana 47907

James T. Hynes, Akihiro Morita, Roberto Bianco, and Brad Gertner

Department of Chemistry and Biochemistry, University of Colorado, Boulder, Colorado 80309

Timothy J. Lee

NASA Ames Research Center, Moffett Field, California 94035

Kirk A. Peterson

Department of Chemistry, Washington State University, Richland, Washington 99352

John H. Seinfeld

Department of Chemical Engineering, California Institute of Technology, Pasadena, California

\section{Financial Support}

DOE, Office of Biological and Environmental Research, Atmospheric Chemistry Program, "Nucleation of Tropospheric Aerosols: A Joint Laboratory and Theoretical Study of Multicomponent Systems" (Garrett and Kathmann)

LDRD project "Oxidation of Volatile Organics Leading to Tropospheric Aerosol Formation" (Dupuis and Kathmann)

NSF, Atmospheric Chemistry," Halogen Chemistry on Stratospheric and Tropospheric Ice" (Gertner, Bianco, Morita, Hynes)

NSF, Atmospheric Chemistry, "Heterogeneous Chemistry on Stratospheric and Tropospheric Sulfate Aerosols" (Bianco, Morita, Hynes)

Number of Hours Allocated for the Past Three Year (Exclude Refund Deposits)

Period: $\quad$ 23-May-98 through 31-May-99

Allocation: $\quad 300,000$ hours on NWmpp1

Period: $\quad$ 1-Jun-99 through 31-May-00

Allocation: $\quad 300,000$ node hours

Period: $\quad$ 1-Jun-00 through 31-May-01

Allocation: $\quad 375,000$ node hours

Number of Hours Actually Used in the Past Year (Exclude Refunded Amounts)

Period: $\quad$ 23-May-98 through 31-May-99

Used: $\quad 300,000$ hours on NWmpp1 

Period:
1-Jun-99 through 31-May-00
Used:
295,194 node hours
Period:
Used:
1-Jun-00 through 31-May-01
212938 node hours

\section{Overview of the Past Three Year's Accomplishments and Activities}

Three primary efforts were undertaken on this project:

- Development of dynamical nucleation theory (DNT) for the calculation of vapor-to-liquid nucleation rates important in the atmosphere,

- Studies of heterogeneous reactions of atmospheric significance,

- Calculation of reaction energetics and reaction rate constants for oxidation of volatile organic compounds in the troposphere.

Overviews of these topics follow.

\section{$\underline{\text { Nucleation of Tropospheric Aerosols }}$}

The main goal of this task is the development of computational methods to calculate rates of vapor-to-liquid nucleation. In DNT nucleation is treated as a kinetic mechanism of cluster growth. The nucleation mechanism is assumed to be adequately described by simple condensation and evaporation of monomers from clusters

$$
A_{1}+A_{i-1} \underset{\alpha_{i}}{\stackrel{\beta_{i}}{\rightleftarrows}} A_{i},
$$

where $\alpha_{i}$ is the evaporation rate constant for loss of a monomer from an $i$-molecule cluster and $\beta_{i-1}$ is the condensation rate constant for addition of a monomer to an (i-1)-molecule cluster. DNT consists of a new theoretical approach, based upon approximate molecular dynamics of the cluster for obtaining the kinetic parameters of the nucleation mechanism $\left(\alpha_{i}\right.$ and $\left.\beta_{i-1}\right)$, followed by solution of the kinetic equations for cluster evolution. The emphasis of DNT is the evaluation of monomer evaporation rate constants. Condensation rate constants are obtained from the evaporation rate constants by detailed balance, which requires calculation of equilibrium constants or populations of clusters in equilibrium with the monomer concentration. The cluster populations are determined from the absolute Helmholtz free energy of a given cluster is a fundamental quantity, as it determines the population of clusters in equilibrium with the monomer concentration. Using detailed balance, the ratio of the evaporation and condensation rate constants is related to differences in Helmholtz free energy between adjacent-sized clusters. This molecular approach entails three subtasks: (a) development of accurate interaction energies for molecules in the cluster needed for molecular simulations; (b) calculation of condensation and evaporation rate constants; and (c) calculation of the steady-state nucleation rate from the kinetic mechanism. The major focus of the work on this project has been on the calculation of condensation and evaporation rate constants. The major computational expense in these rate constant calculations is the calculation of Helmholtz free energies for forming $i$-molecule clusters that are constrained within spherical shells of fixed radii. The initial calculations were performed for nucleation in supersaturated water vapor, for which the nucleation is controlled by clusters including up to tens of molecules. Molecular simulations of the Helmholtz free energies for 
these size clusters are computational intensive and benefit greatly from access to NWMPP1. To perform these calculations, a new Monte Carlo simulation code was implemented in parallel on the MPP during the first year of the project. During the second year of the project we learned a significant amount about the extreme sensitivity of computed nucleation rates to the evaporation and condensation rate constants, which guided further development of computational tools.

For small cluster, the rate constants for evaporation are typically much larger than those for condensation so that the formation of larger clusters is unlikely and cluster populations decrease as a function of increasingly cluster size in this size range. As the cluster grow, condensation becomes faster, while evaporation decreases, until they are approximately equal. The cluster size at which the forward and backward rates are about equal is called the critical nucleus. This is the cluster with the smallest population. Small changes in kinetic parameters can shift the critical cluster size and greatly alter the nucleation rate. Given the delicate nature of the nucleation process it is important to assess the effects of uncertainties in the kinetic parameters that govern the steady-state nucleation rate. Sensitivity analysis provides insight into the influence and identity of the dynamical quantities to which the nucleation rate, $J$, is most sensitive. This, in turn, aids in the elucidation of specific reaction mechanisms and reduces computational effort by focusing only on those clusters that affect the nucleation rate. The sensitivity analysis can be performed in two ways. (1) When the nucleation rate is expressed as a function of the evaporation and condensation rate constants $\left(\alpha_{i}\right.$ and $\left.\beta_{i}\right), J=J(\alpha, \beta)$, then the nucleation rate has nearly unit sensitivity to all pre-critical clusters (e.g., a factor of 2 change in one of the kinetic parameters results in a factor of 2 change in the nucleation rate). (2) When the nucleation rate is expressed as a function of the condensation rate constants and equilibrium cluster populations $\left(\mathrm{N}_{i}^{\mathrm{EQ}}\right), J=J\left(\beta, \mathrm{N}^{\mathrm{EQ}}\right)$, then the sensitivity is peaked about the critical cluster. Thus, if the condensation rate constants and equilibrium cluster populations (or equivalently the absolute Helmholtz free energies) of the clusters around the critical size can be determined directly, then the sensitivity of the nucleation rate on all the pre-critical clusters can be minimized. Calculations of equilibrium constants needed in the detailed balance expression require determination of the differences of Helmholtz free energies between adjacent clusters. Absolute free energy of the $i$-cluster can be obtained by accumulating free energy differences between adjacent clusters

$$
\mathrm{A}_{i}=\Delta \mathrm{A}_{2,1}+\Delta \mathrm{A}_{3,2}+\ldots+\Delta \mathrm{A}_{i, i-1}
$$

However, uncertainties for each free energy difference are also accumulated so the uncertainty in the absolute free energy $\mathrm{A}_{i}$ contains uncertainties from all pre-critical clusters. To minimize sensitivity to the parameters of the pre-critical clusters, we need a method to directly calculate the absolute free energy of an $i$-cluster, which is computational much more demanding. The major focus of the second year was the development of new computational tools, based upon the external work technique ${ }^{1}$ to directly calculate the absolute free energies of clusters near the critical cluster. In the external work technique an estimate of the absolute Helmholtz free energy is provided by determination of the external work $\mathrm{W}$. The change in internal energy of a system $\Delta \mathrm{E}$ for a given process is given by $\Delta \mathrm{E}=\mathrm{Q}+\mathrm{W}$, where $\mathrm{Q}$ is the heat absorbed by the system. For a reversible process the change in Helmholtz free energy of the system $\Delta \mathrm{A}$ is equal to the work

1 W. P. Reinhardt and J. E. Hunter, J. Chem. Phys. 97, 1599-1601 (1992); J. E. Hunter, W. P. Reinhardt and T. F. Davis, J. Chem. Phys. 99, 6856-6864 (1993) 
done on the system. For an irreversible process $\mathrm{W} \geq \Delta \mathrm{A}$. For calculating the absolute free energy of an $i$-molecule water cluster, we calculate the work for the process of 'turning on' the interaction potential between molecules in the cluster. The potential energy surface is slowly changed from noninteracting (an ideal gas state) to a fully interacting potential, the total heat released to the bath is accumulated during this process, and the change in internal energy $\Delta \mathrm{E}=\mathrm{E}$ (final) $-\mathrm{E}$ (initial) is calculated. The external work is then determined by $\mathrm{W}=\Delta \mathrm{E}-\mathrm{Q}$, which provide an upper bound to the free energy difference between the fully interacting and ideal gas states. The absolute free energy of the ideal gas state is obtained from an analytical expression, thereby allowing determination of the absolute free energy of the interacting state. A lower bound to the free energy is obtained by reversing the process (starting with the interacting state and slowly changing to the ideal gas state). A Monte Carlo code was developed to perform external work calculations on water clusters and a parallel version of this program was implemented in the third year of the project.

Our primary focus for the third year of this project was the extreme sensitivity of the nucleation rate to kinetic parameters. Since the kinetic parameters are sensitive to the underlying potential energy surface (PES) of the system, the nucleation rate also exhibits significant sensitivity to the PES. Successful implementation of the external work technique allowed us to calculate free energy differences for clusters with difference potentials. All our previously reported calculations on water clusters have been performed using the Dang-Chang polarizable water model. We have initiated calculations of free energy and evaporation rate constants for other PESs. For example, we have seen large changes in the evaporation rate constants and Helmholtz free energies for water clusters with $i=2-6$ when the TIP4P potential is used instead of the Dang-Chang potential.

Molecular simulations of equilibrium statistical mechanical properties (such as the Helmholtz free energy of cluster formation) required accurate molecular interaction potentials. For the molecular simulations performed in water clusters, the rigid, polarizable potential of Dang and Chang was used. To understand the accuracy of this potential for calculating the volume dependence of the cluster Helmholtz free energies, benchmark ab initio calculations of water clusters were performed for comparison with the Dang-Change potential. One major objective of this project is to develop a molecular-level theoretical formulation of aerosol nucleation that demonstrates an understanding of the factors controlling aerosol formation in binary and ternary systems (e.g., systems containing two and three nucleating components). With this objective in mind, we have extended DNT to multicomponent systems in the past year implemented the extended theory in the simulation codes. Benchmark ab initio electronic structure calculations were also carried out for sulfuric acid (SA)-water, ammonia-water and SA-ammonia to aid in the development of accurate interaction potentials, which are needed in studies of nucleation in binary and ternary systems involving SA, ammonia, and water. Work has also been proceeding to fit a polarizable ammonia-water potential.

\section{Heterogeneous Reactions of Atmospheric Significance}

Sulfate aerosols are supercooled liquid concentrated aqueous solutions of sulfuric acid. It has long been recognized that the hydrolysis reaction of dinitrogen dioxide N2O5 with water to produce nitric acid, occurring on sulfate aerosols, is the key heterogeneous reaction in the 
midlatitude stratosphere relevant to the stratospheric ozone depletion. Sulfate aerosols are also of significance in the troposphere. Neither the surface structure of these aerosols nor the reaction mechanism is currently understood.

In the first year of the project, we began to characterize the bulk and surface structures of a supercooled sulfate aerosol at low temperature (about 220K) using molecular dynamics (MD) simulations, and electronic structure calculations. These results were utilized to evaluate (1) equilibrium structure (density and concentration profiles, etc.) near the surface, and (2) free energy of proton transfer between sulfur species and water in order to initially assess the acidity conditions at the aerosol surface. It is a key feature that proton transfer reactions can occur in the surface region, so that the ionization state of the surface is not known a priori; this ionization state is expected to be crucial for the $\mathrm{N} 2 \mathrm{O} 5$ hydrolysis reaction mechanism and rate. A new MD program code was developed for the MPP1 parallel computers, where independent MD trajectories were generated on the parallel nodes having different initial conditions.

Unfortunately, we were unable to convince ourselves that we could reliably predict the microscopic geometric structure and ionization state of a sulfate aerosol surface using the methods above. The essential difficulty is that the free energy of proton transfer between a sulfur species and a water molecule (e.g. between molecular sulfuric acid $\mathrm{H}_{2} \mathrm{SO}_{4}$ and water to produce the hydrogen sulfate ion $\mathrm{HSO}_{4}{ }^{-}$and the hydronium ion $\mathrm{H}_{3} \mathrm{O}^{+}$) which is key to deciding the surface ionization state, depends sensitively on the ionization state in the rest of the surface, which is unknown and ultimately must be determined in some self-consistent way. In short, while we were able to predict the surface structures of a model sulfate aerosol with a given fixed surface ionic composition (and suggest reaction mechanisms on such surfaces), we could not confidently predict the surface's ionic character or its structure in an a priori fashion.

Accordingly, in the second year of the project, we turned to a different way to gain information about the sulfate aerosol surfaces. This was via consideration of Sum Frequency Generation (SFG) spectroscopy. The surface-sensitive SFG nonlinear optical spectroscopy technique, which allows the examination of a surface vibrational spectrum, is an especially important tool for the study of liquid-vapor interfaces, where many high vacuum techniques suitable for solid surfaces cannot be employed. SFG experiments had already been done on laboratory model sulfate aerosol surfaces, with conflicting interpretations. Our concept was to generate different fixed surface structures of a sulfate aerosol, exploring a range of surface ionization states-which we are perfectly able to do with the methods described above - to compare with the experiments and thus infer the correct surface structure. However, connecting an observed SFG signal to the underlying molecular structure is not at all simple, and itself presented a major theoretical/computational challenge which had to be met for the technique to provide the desired molecular level window on the surface structure.

We thus began the construction of a theory for SFG by developing a first generation theoretical description of the SFG spectrum which includes the requisite microscopic hyperpolarizability tensors and their dependence on molecular vibrations, for the water surface, using a combination of electronic structure calculations and molecular dynamics simulations. The direct simulation of the vibrations was finessed via a separate theoretical analysis of the frequency shifts, dipole moment and polarizability vibrational coordinate derivatives, etc., and certain simplifying 
approximations were made. Analysis of the resulting SFG spectrum of water, which was in encouraging overall good agreement with experiment, established the (different) degree of surface sensitivity for different $\mathrm{OH}$ stretching vibration regions, and revealed a number of surprising microscopic features, e.g., that the 'dangling bond' $\mathrm{OH}$ portion of the spectrum usually thought of as arising exclusively from water $\mathrm{OH}$ bonds perpendicular to the surface, and hence free of any hydrogen bonding to the $\mathrm{H}$ - in fact has considerable contribution from $\mathrm{OH}$ bond configurations significantly tilted from the surface normal.

But despite this considerable success, it was clear that further improvements were definitely required. Thus, for example, application of the theory to a water/methanol mixture, where the surface structure is already well characterized, succeeded only qualitatively in reproducing the observed behavior of the SFG OH dangling bond signal. Thus, improvements to the theory were very definitely required if one were to have a tool capable of reliably translating a microscopic surface structure into an expected SFG spectrum.

In the third year, we improved and greatly extended our first generation SFG description, to create a new, second-generation theory, based on a dynamical, time dependent approach. The new theory avoided some assumptions and approximations involved in our former theory concerning the modeling of the frequency-dependent molecular hyperpolarizability and its perturbation in a condensed environment, and thus should be much more robust and widely applicable than the former one. We have applied it to the water surface, and find reasonably good agreement with experiment, although some discrepancies remain. After the performance of the new theory is extensively checked (e.g. via the methanol/water surface), it will be applied in mixed electronic structure/molecular dynamics simulations to interpret the SFG spectra of stratospheric sulfate aerosols, using the strategy we outlined above. This extensive checking stage is currently underway. In addition, we have been exploring a separate and complementary strategy of examining smaller cluster systems via electronic structure calculations to gain some insights on the aerosol surface structures and possible reaction mechanisms on them.

Reaction Energetics and Rate Constants for Oxidation of Volatile Organic Compounds Important in the Troposphere

Radical reactions are difficult to study both experimentally and theoretically. Experimentally, radical reactions are very fast, complicating the identification of reaction pathways and intermediates. Theoretically, an accurate description of the low-energy barriers requires sophisticated treatment of electron correlation. The test reaction $\mathrm{OH}+\mathrm{C}_{2} \mathrm{H}_{4}$ was chosen because (1) of its simplicity and (2) there exists experimental rate constants for comparison. Initially, calculations were performed at the MP2//cc-pvdz level that gave a barrier of $7.5 \mathrm{kcal} / \mathrm{mol}$ and a resulting rate constant that was eleven orders of magnitude below experiment. Using the optimized geometries at the MP2 level single point calculations were carried out at the UCCSD(T)//avdz level. The resulting energetics resulted in the barrier being reduced to $-1 \mathrm{kcal} / \mathrm{mol}$ (i.e., below the energy of reactants); however, the rate constant was too slow by an order of magnitude and the temperature dependence was in the wrong direction compared to experiment. Since optimization of the reaction path at the UCCSD(T) level is intractable, the use of the DFT with the MPW1K functional of Truhlar and coworkers was explored. The resulting reaction path had the correct negative barrier with respect to reactants by $-1.9 \mathrm{kcal} / \mathrm{mol}$ and the variational 
transition state theory (VTST) estimate for the rate agrees well with experiment: $k^{\mathrm{VTST}}=6.3 \times 10$ ${ }^{-12} \mathrm{cc} / \mathrm{molecule} / \mathrm{s}$ at $300 \mathrm{~K}$ compared to $k^{\mathrm{EXP}}=\left(4.5 \times 10^{-12}\right.$ to $\left.9.9 \times 10^{-12}\right) \mathrm{cc} / \mathrm{molecule} / \mathrm{s}$. This reaction goes through a van der Waals complex $(-3.2 \mathrm{kcal} / \mathrm{mol})$, through a first transition state ($1.9 \mathrm{kcal} / \mathrm{mol})$, into a $2^{\text {nd }}$ complex $(-2.2 \mathrm{kcal} / \mathrm{mole})$, through a $2^{\text {nd }}$ transition state $\left(\sim 5 \times 10^{-3}\right.$ $\mathrm{kcal} / \mathrm{mole}$ above the $2^{\text {nd }}$ complex $)$, and then to the product $\mathrm{C}_{2} \mathrm{H}_{4} \mathrm{OH}(-33.6 \mathrm{kcal} / \mathrm{mol})$ as shown schematically in the figure. Furthermore, the theoretical rate constants are consistent with the measured temperature dependence. These results represent the most comprehensive study of $\mathrm{OH}$ $+\mathrm{C}_{2} \mathrm{H}_{4}$ reaction energetics to date. A previous investigation of this reaction by J. Villa et al. (J. Chem. Phys., 107, 7266, (1997)) considered only $0.5 \AA$ along the reaction path and thus completely missed both the $2^{\text {nd }}$ complex and $2^{\text {nd }}$ transition state. Calculations are underway to benchmark these latest results against those at the UCCSD(T) level of theory. In addition, calculations for the stationary points along the reaction path for $\mathrm{OH}+$ butadiene are currently being performed.

\section{List of Publications Resulting From This Work}

G. K. Schenter, S. M. Kathmann, and B. C. Garrett, "Dynamical Nucleation Theory: A New Molecular Approach to Vapor-Phase Nucleation," Phys. Rev. Lett. 82, 3484 (1999).

G. K. Schenter, S. M. Kathmann, and B. C. Garrett, “A Variational Transition State Theory of Nucleation and its Relation to Physically Consistent Clusters," J. Chem. Phys. 110, 7951 (1999).

S. M. Kathmann, G. K. Schenter, and B. C. Garrett, "Dynamical Nucleation Theory: Calculation of Condensation Rate Constants for Small Water Clusters," J. Chem. Phys. 111, 4688-4697 (1999).

S. M. Kathmann, G. K. Schenter, and B. C. Garrett, "Dynamical Nucleation Theory," invited contribution to Frontiers in Science and Technology. (IAPS Press, La Jolla, CA).

S. M. Kathmann, G. K. Schenter, and B. C. Garrett, "Dynamical Nucleation Theory," in Nucleation and Atmospheric Aerosols 2000, ed. B. N. Hale and M. Kulmala, (American Institute of Physics, Melville, New York, 2000), p. 197.

B. C. Garrett, S. M. Kathmann, and G. K. Schenter, "Kinetics of Cluster Evaporation and Condensation Important in Homogeneous Vapor Phase Nucleation," in Nucleation and Atmospheric Aerosols 2000, ed. B. N. Hale and M. Kulmala, (American Institute of Physics, Melville, New York, 2000), p. 201.

A. Morita and J. T. Hynes, "A Theoretical Analysis of the Sum Frequency Generation Spectrum of the Water Surface," Chem. Phys. 258, 371 (2000). 


\section{List of Presentations Resulting From This Work}

$\underline{\text { Invited presentations }}$

B. C. Garrett, "A Molecular Approach to Vapor-to-Liquid Nucleation: Simulations of Evaporation and Condensation of Molecular Clusters," Chemistry Department, University of British Columbia, Vancouver, British Columbia, February 5, 1999.

B. C. Garrett, "A Molecular Approach to Vapor-to-Liquid Nucleation: Simulations of Evaporation and Condensation of Molecular Clusters," Chemistry Department, University of Washington, Seattle, Washington, April 14, 1999.

S. M. Kathmann, "A Molecular Approach to Vapor-to-Liquid Nucleation: Simulations of Evaporation and Condensation of Molecular Clusters," Environmental Molecular Sciences Symposia and First Users' Meeting, Pacific Northwest National Laboratory, Richland, Washington, July 1999.

S. M. Kathmann, "Dynamical Nucleation Theory: Application to Small Water Clusters," Frontier-Science Research Conference on Aerosol Science and Technology, La Jolla International School of Science, The Institute for Advanced Physics Studies, La Jolla, California, August 16-19, 1999.

G. K. Schenter, "Variational Transition State Theory of Vapor Phase Nucleation,” FrontierScience Research Conference on Aerosol Science and Technology, La Jolla International School of Science, The Institute for Advanced Physics Studies, La Jolla, California, August 16-19, 1999.

SS Xantheas, "Critical Issues in the Understanding and Modeling of Collective Effects in Water", Symposium on Water and Water Clusters, Physical Chemistry Division, American Chemical Society National Meeting, New Orleans, LA, August 22-26, 1999.

J. T. Hynes and A. Morita, "Molecular modeling of sulfate aerosols: Surface and bulk structures of concentrated supercooled sulfuric acid solutions", in surface chemistry section honoring G. A. Somorjai, 219th ACS meeting at San Francisco, March 26-30, 2000.

B. C. Garrett, "Dynamical Nucleation Theory: A New Molecular Approach to Modeling Vaporto-Liquid Nucleation," Symposium on New Directions in Chemical Modeling, Physical and Theoretical Chemistry Division, Canadian Society for Chemistry Conference, Calgary, Alberta, May 27-31, 2000.

B. C. Garrett, "Thermochemistry and Kinetics of Small Water Clusters," Workshop on Water in Confined Geometries: $\mathrm{H}_{2} \mathrm{O}$ Clusters, Particles, Surfaces and Thin Layers, Telluride, Colorado, July 30 - August 5, 2000. 
A. Morita and J. T. Hynes, "Theory and Simulation of Sum Frequency Generation Spectra for Aqueous Interfaces", CNRS Groupe de Recherches, Porquerolles, France, September 2000.

B. C. Garrett, "Dynamical Nucleation Theory: A New Molecular Approach to Modeling Vaporto-Liquid Nucleation," Chemistry Department, The Ohio State University, Columbus, Ohio, January 29, 2001.

B. C. Garrett, "A Variational Transition State Theory Approach to Cluster Evaporation and Condensation Important in Homogeneous Vapor-Phase Nucleation," Chemical Dynamics Symposium in Honor of William H. Miller, Berkeley, California, March 29-31, 2001.

B. C. Garrett, "A Variational Transition State Theory Approach to Cluster Evaporation and Condensation Important in Homogeneous Vapor-Phase Nucleation," Symposium on Quantum Engineering at Conference on "Applied Statistical Physics. Molecular Engineering" (ASTATPHYS-MEX-2001) Cancun, Mexico, July 22-27, 2001.

A. Morita, "Structure and Dynamics at liquid-vapor interfaces relevant to the heterogeneous atmospheric chemistry", in 10th Symposium on Theoretical Chemistry, Seto, Japan, August 2-5, 2000 .

A. Morita, "Theory of sum frequency generation spectroscopy for liquid-vapor interfaces", in Supercomputer Workshop, Institute of Molecular Science, Okazaki, Japan, March 22-23, 2001.

A. Morita, "Theoretical analysis of sum frequency generation spectra of water surface", Department of Molecular Engineering, Kyoto University, Japan, June 15, 2001.

\section{Contributed Presentations (Oral and Poster)}

B. C. Garrett, "Nucleation of Tropospheric Aerosols: A Joint Laboratory and Theoretical Study of Multicomponent Systems," Annual Science Meeting of the Atmospheric Chemistry Program, Office of Biological and Environmental Research, Department of Energy, Falls Church, Virginia, February 23-25, 1999.

B. C. Garrett, "Dynamical Nucleation Theory: A New Molecular Approach to Vapor-Phase Nucleation," American Physical Society National Meeting, Atlanta, Georgia, March 22-26, 1999.

B. C. Garrett, "Nucleation of Tropospheric Aerosols: A Joint Laboratory and Theoretical Study of Multicomponent Systems," Gordon Research Conference on Atmospheric Chemistry, Newport, Rhode Island, June 13-18, 1999.

S. M. Kathmann, "Dynamical Nucleation Theory: A New Molecular Approach to Vapor-Liquid Nucleation," Northwest Regional Meeting of the American Chemical Society, Portland, Oregon, June 21-23, 1999. 
S. M. Kathmann, "Dynamical Nucleation Theory: Calculation of Evaporation Rate Constants for Water Clusters," American Conference on Theoretical Chemistry, Boulder, Colorado, June 28July 2, 1999.

B. C. Garrett, "Thermochemistry and Kinetics of Small Water Clusters," Symposium on Water and Water Clusters, Physical Chemistry Division, American Chemical Society National Meeting, New Orleans, LA, August 22-26, 1999.

S. M. Kathmann, "Dynamical Nucleation Theory," 15th International Conference on Nucleation and Atmospheric Aerosols, Rolla, Missouri, August 6-11, 2000.

B. C. Garrett, "Kinetics of Cluster Evaporation and Condensation Important in Homogeneous Vapor Phase Nucleation," 15th International Conference on Nucleation and Atmospheric Aerosols, Rolla, Missouri, August 6-11, 2000.

B. C. Garrett, "Thermochemistry and Kinetics of Small Water Clusters," Symposium on Solvated Molecules and Ions: from Clusters to Condensed Phases, Pacifichem 2000, Honolulu, Hawaii, December 14-19, 2000.

A. Morita and J. T. Hynes, "Theoretical analysis of sum frequency generation spectra for liquidvapor interfaces", in 11th Symposium on Atmospheric Chemistry, Toyohashi, Japan, Jan. 11-12, 2001.

A. Morita and J. T. Hynes, "Theoretical analysis of sum frequency generation spectra for water surface”, in Computational Science Workshop 2001, Tsukuba, Japan, March 11-13, 2001.

A. Morita and J. T. Hynes, "New theoretical analysis of sum frequency generation spectroscopy”, in 7th Atmospheric Chemistry Workshop, Ohtsu, Japan, May 30-June 1, 2001.

A. Morita and J. T. Hynes, "Theoretical analysis of sum frequency generation spectra for liquidvapor interfaces”, in 222nd ACS Symposium, Chicago IL, August 26-30, 2001.

\section{$\underline{\text { List of Significant Methods/Routines or Codes }}$}

Metropolis Monte Carlo Simulation of Cluster Helmholtz Free Energies

A Monte Carlo code was written to perform external work calculations on water clusters and was adapted to run on any number of nodes on the MPP. The Monte Carlo code utilizes intrinsic commands that specify which node it is on. Given this node number an initial random configuration of water molecules is generated. Jobs are submitted to the MPP using a queuing system, which requires a batch script. The batch script specifies the following: the total number of nodes to be used, to what directories to write the output, how much time will be required for job completion, from what account to charge the computer time, on what nodes to place the executables, and to erase all files on each node after completion. The resulting data sets are combined and averaged. By using many different starting configurations a thorough sampling of the reversible paths which join the two thermodynamic states of interest can be achieved. For a 40 water mole- 
cule cluster with 10 initial starting configurations run for 3 million Monte Carlo steps at $\mathrm{T}=243$ $\mathrm{K}$, the Helmholtz free energy difference is $\Delta \mathrm{A}=\mathrm{A}-\mathrm{A}^{\text {ideal }}=(-146.7 \pm 3.1) \mathrm{kcal} / \mathrm{mol}$.

Theoretical Analysis Method of the SFG Spectral Based on MD Simulations

This program has been developed to perform the theoretical analysis of SFG spectra. It requires MD trajectories as input, and relates the molecular orientations to the frequency-dependent hyperpolarizability for obtaining the macroscopic nonlinear susceptibility. The MD trajectories are generated by a parallel MD program also developed by our group. 


\section{Appendix A - Full report of First Year Activities and Accomplishments}

\section{$\underline{\text { Nucleation of Tropospheric Aerosols }}$}

We have developed a new theoretical approach to homogeneous gas-to-vapor nucleation that goes well beyond the classical theory of nucleation and also removes approximations of other molecular theories. We adopt the usual starting point for molecular approaches, which is the assumption that addition and removal of monomers from clusters adequately describes the nucleation kinetics. The nucleation rate is obtained by solving the pseudo-first order kinetic equations (assuming the monomer concentration of the nucleating species is large compared to the cluster concentrations and can be approximated as a constant). The first major approximation in other molecular theories is that the condensation rate constant can be approximated by the collision rate of monomers with the cluster. This formulation for the condensation rate constant approximates the cross section for collisions by the surface area of a sphere of radius $r$, and assumes unit sticking probability upon collision. The evaporation rate constant is then evaluated using detailed balance and the task then reduces to evaluating the equilibrium cluster distribution or equivalently the free energies for forming the clusters from monomers. Because the clusters that are important in the nucleation process can dissociate, the equilibrium cluster distribution or Helmholtz free energy exhibits a dependence on the constraining volume and actually diverges as the volume containing the cluster increases. Treatment of the volume dependence of this free energy has been a source of confusion and controversy in the development of a general molecular theory of nucleation.

In contrast to previous theories, our new molecular theory of nucleation provides a method for directly calculating the evaporation and condensation rate constants. This approach has two major advantages over the previous molecular approaches. First, the direct evaluation of the rate constants avoids approximating the condensation rate constant using a simple gas collision rate. Second, the formulation of the rate constants naturally yields a consistent prescription for dealing with the volume dependence of the free energy for cluster formation and actually exploits this dependence.

We view the processes of evaporation and condensation as gas-phase dissociation and association reactions. This view of the nucleation process as a gas-phase reaction mechanism composed of dissociation and association reactions is quite different than the perspective generally encountered in the nucleation literature where nucleation is viewed as density fluctuations in a supersaturated fluid. Given the large distance between water monomers and clusters in the vapor $\left(>10^{4}-10^{5} \AA\right.$ for water at a supersaturation of 5), the view of nucleation as a gas-phase reaction mechanism is quite reasonable. Transition state theory (TST) provides a convenient framework to evaluate equilibrium rate constants for these kinetic processes.

The TST rate constant is approximated by the equilibrium one-way flux through a dividing surface separating reactants from products. For evaporation of a monomer from an i-molecule cluster, reactants are an i-molecule cluster and products are a monomer infinitely separated from an (i-1)-molecule cluster. In the present work, we choose the dividing surface to be a sphere with its center at the center of mass of the i-molecule cluster; the radius of the sphere then 
determines the location of the dividing surface. (Spherical dividing surfaces are chosen to make contact with previous work that used spherical constraining volumes as well as the simplicity is lends to the theory.) For the dissociation reaction studied here, the location of the dividing surface is guided by variational TST (VTST). The fundamental assumption of TST guarantees that the TST approximation to the classical reactive flux is greater than or equal to the exact classical flux. This upper bound is the basis for variational TST in which the transition state dividing surface is located to minimize the reactive flux. Thus, VTST gives a prescription for defining a unique location of the dividing surface (or radius of the sphere) for each reaction.

In the new theory the rate constant for monomer evaporation from an i-molecule cluster is given by

$$
\mathrm{k}_{\mathrm{i}, \text { evap }}^{\mathrm{VTST}}(\mathrm{T})=-\left.\left(\frac{1}{2 \pi \mathrm{mk}_{\mathrm{B}} \mathrm{T}}\right)^{1 / 2} \frac{\mathrm{d}}{\mathrm{dr}} \mathrm{A}_{\mathrm{i}}(\mathrm{T}, \mathrm{r})\right|_{\mathrm{r}=\mathrm{r}_{\mathrm{i}}^{\mathrm{a}}}
$$

where $\mathrm{m}$ is the monomer mass, $\mathrm{k}_{\mathrm{B}}$ is Boltzmann's constant, $\mathrm{T}$ is temperature, and $\mathrm{A}_{\mathrm{i}}(\mathrm{T}, \mathrm{r})$ is the Helmholtz free energy for forming an i-molecule cluster in a sphere of radius $r$. The value of $r$ that minimizes the rate constant is denoted $r_{i}^{a ̀ ~}$. It is worth noting that free energy curves $A_{i}(T, r)$ have been computed by several groups dating back to the early 1970s, but it is only our work within the last year that has shown the evaporation rate constant can be related to the derivative of $\mathrm{A}_{\mathrm{i}}(\mathrm{T}, \mathrm{r})$ with respect to $\mathrm{r}$. Once the evaporation rate constant is obtained, the condensation rate constant can be obtained (for monomer addition to an (i-1)-cluster) using detailed balance. The resulting expression for the condensation rate requires evaluation of the absolute value of the Helmholtz free energy, whereas the evaporation rate constant requires its derivative with respect to $\mathrm{r}$.

The absolute value of the free energy and its derivative are obtained by Monte Carlo simulations. Accurate calculations of $\mathrm{A}_{\mathrm{i}}(\mathrm{T}, \mathrm{r})$ vs. $\mathrm{r}$ require extensive simulations that sample configurations with water molecules evaporated (or with their center of mass at least 4- $6 \AA$ from the nearest neighbor's center of mass) as well as those in which all water molecules are hydrogen bonded together. The configuration space of the i-molecule cluster is sampled in an extended canonical ensemble in which the volume is allowed to fluctuate under imposed constant pressure and constant temperature using Metropolis Monte Carlo. During the course of the Monte Carlo simulation over configurations and volume, the range or volume (or r) is partitioned into bins for accumulation of population. The relative probability of a cluster forming within a given volume $\mathrm{v}$ (or spherical radius $\mathrm{r}$ ) is obtained from the normalized population and the relative value of the Helmholtz free energy is obtained from the probability. NWMPP1 provides an excellent platform on which to perform these simulations since the total sampling can be split up over many processors. In order to obtain good statistics many independent simulations are performed and the results from each simulation averaged. A Monte Carlo code was adapted to run $\mathrm{N}$ independent simulations on $\mathrm{N}$ nodes on the MPP1, where in practice values of $\mathrm{N}=64,128$, or 256 were typically used. Each simulation is initialized with the total volume set to the maximum volume allowed in the simulation and with a configuration that randomly fills only a fixed portion of the available total volume. To insure that the $\mathrm{N}$ simulations are indeed independent, the initial fixed portion of the total volume for each simulation was uniquely chosen (based upon the node 
number for that simulation) such that the initial configurations for all $\mathrm{N}$ simulations spanned the space of possible structures from compact ones to disperse ones with i weakly interacting molecules. Each simulation begins with a large number of thermalization steps in which no statistics are accumulated. Typically the thermalization takes only a small percentage of the total run time, but as $\mathrm{N}$ increases the total sampling points per simulation decreases while the thermalization period stays constant, so the percentage not being used in sampling increases. This is the major cause of nonlinear scaling with processors; however, as seen in Figure 1, the scaling remains quite good up to 440 nodes.

One difficulty with the MPP implementation was the observed need for sufficiently long simulations to insure that disperse configurations were sampled In practice, during the thermalization process, the more disperse initial configurations tend to condense, since the more compact structures are energetically favored. Thus, even though many of the simulations are initialized with disperse configurations, by the time statistics are accumulated the configurations are compact. Adequate sampling of the less probable disperse configurations requires larger single sample sizes rather than a larger number of smaller sample sizes. This also limited the effectiveness of our use of the MPP.

Results of the simulation for water clusters with 2 to 10 molecules are presented in Figure 2. The Helmholtz free energies appear to remain relatively constant over a range of $r$, particular for the larger clusters. Even so, the derivatives of these curves exhibit well-defined minima from which the evaporation rate constants are calculated. Table I displays the optimum $r$ value $r_{i}^{\grave{a}}$, absolute value of the Helmholtz free energy at $r_{i}^{\mathrm{a}}$, and the evaporation rate constant obtained from these calculations.

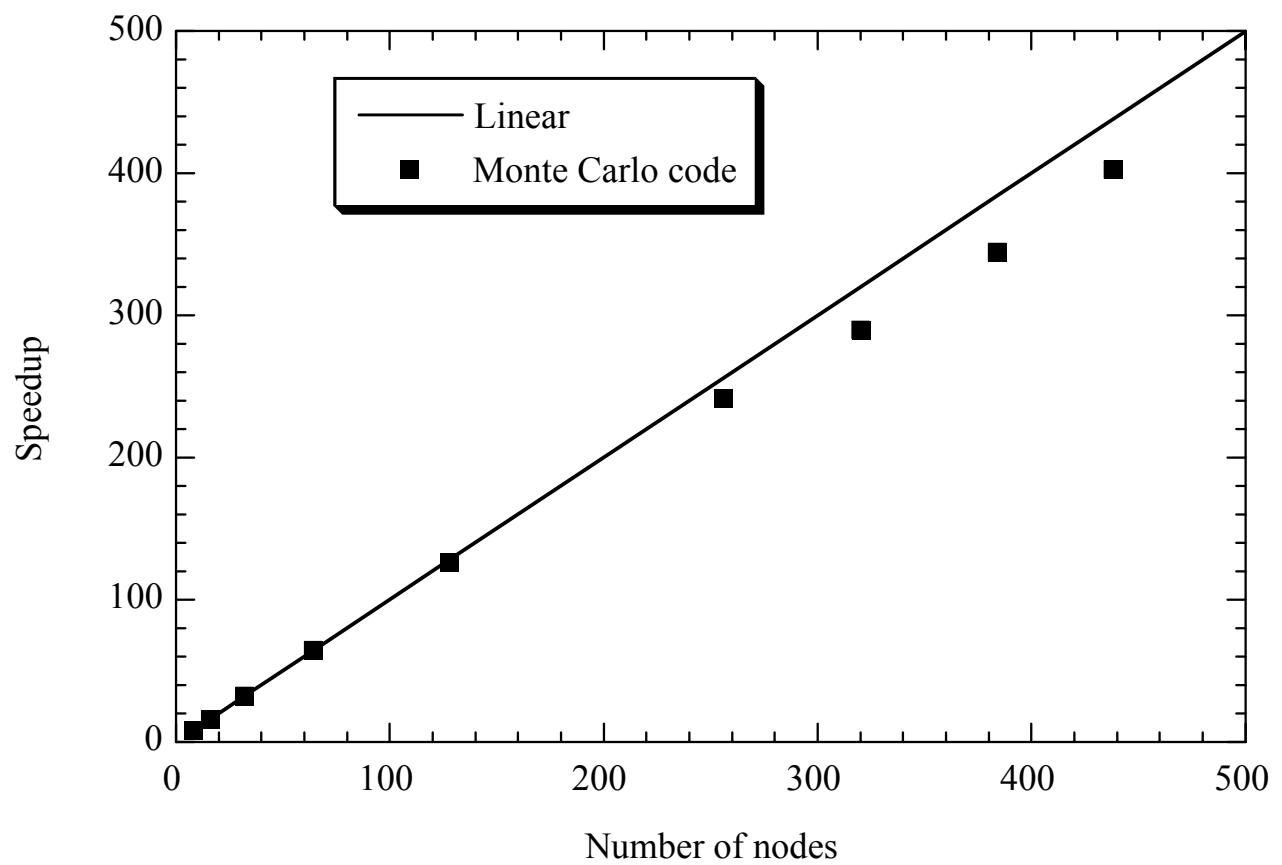

Figure 1. Speed up of the Monte Carlo simulation code on NWMPP1 


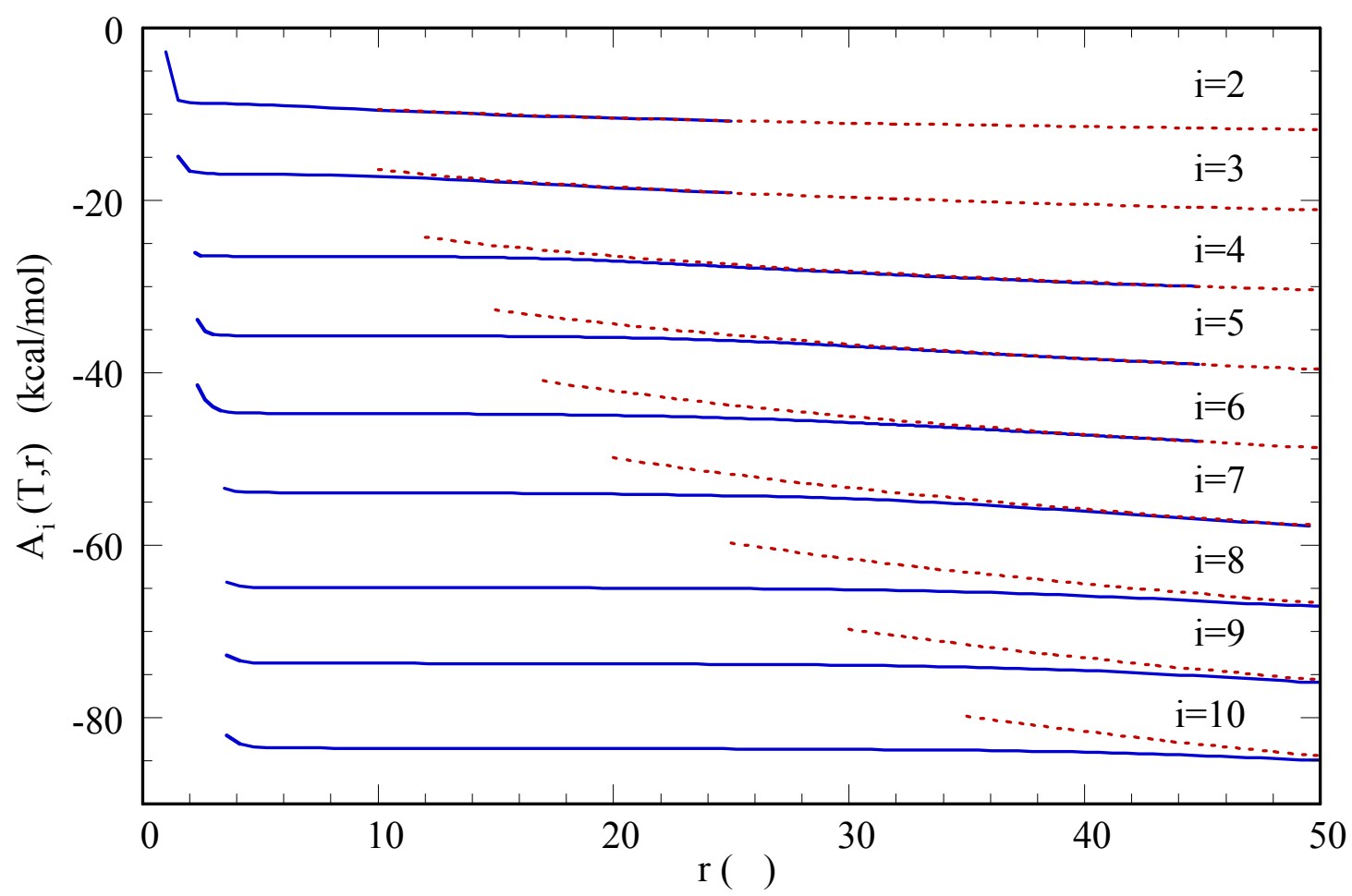

Figure 2. Helmholtz free energy as a function of radius of constraining sphere for i-molecule water clusters at $243 \mathrm{~K}$. The polarizable potential of Dang and Chang was used in the simulations. Solid curves are the results of simulations using the potential of Dang and Chang and the dashed curves are the analytical results for noninteracting molecules (i.e., the ideal gas limit).

Table I. A comparison of the radii $\mathrm{r}_{\mathrm{i}}^{\mathrm{a}}$, Helmholtz free energies A(T, $\left.\mathrm{r}_{\mathrm{i}}^{\grave{a}}\right)$, and VTST rate constant $\mathrm{k}_{\mathrm{evap}, \mathrm{i}}^{\mathrm{VTST}}(\mathrm{T})$ for the $\mathrm{i}$-molecule water clusters at $243 \mathrm{~K}$.

\begin{tabular}{rrcc}
\hline $\mathrm{i}$ & $\mathrm{r}_{\mathrm{i}}^{\mathrm{a}}(\AA)$ & $\mathrm{A}\left(\mathrm{T}, \mathrm{r}_{\mathrm{i}}^{\mathrm{a}}\right)(\mathrm{kcal} / \mathrm{mol})$ & $\mathrm{k}_{\text {evap }, \mathrm{i}}^{\mathrm{VTST}}(\mathrm{T})\left(10^{9} \mathrm{~s}^{-1}\right)$ \\
\hline 2 & 2.8 & -8.8 & 160 \\
3 & 4.3 & -17.0 & 49 \\
4 & 5.8 & -26.5 & 4.7 \\
5 & 5.8 & -35.7 & 9.9 \\
6 & 6.3 & -44.9 & 13 \\
7 & 6.8 & -54.3 & 9.4 \\
8 & 7.5 & -63.9 & 7.2 \\
9 & 8.0 & -73.7 & 5.3 \\
10 & 8.5 & -83.6 & 4.6 \\
\hline \hline
\end{tabular}


The development of accurate potential energy functions (PEF) is a crucial task in this work. Because we are interested in describing clusters as few as two molecules up to tens of molecules, it is necessary to have PEFs that can reproduce properties of small clusters and continue to the correct limit of bulk phase properties as the clusters grow. The development of accurate PEFs requires benchmark studies interaction energies to aid in their construction and to test their accuracy. In addition, benchmark studies for clusters with two to several molecules are needed to allow critical tests of how well many body effects are included in the PEFs.

During the first year of this project we have established accurate energetics for the $\mathrm{NH}_{3}-\mathrm{NH}_{3}$ and $\mathrm{NH}_{3}-\mathrm{H}_{2} \mathrm{O}$ interactions as well as a measure of the cooperative effects in the ammonia trimer. The interactions between two ammonia molecules as well as between $\mathrm{NH}_{3}$ and $\mathrm{H}_{2} \mathrm{O}$ were estimated at the second order Møller-Plesset (MP2) level of theory with the family of augmented correlation consistent basis set of Dunning and co-workers. Estimates of the correction due to basis set superposition error (BSSE) and higher order of electron correlation at the CCSD(T) level of theory were also taken into account. The NWCHEM suite of programs was used in order to optimize the structure of the clusters and perform a vibrational analysis at the resulting minimum energy configurations.

We have revisited the long-standing issue of the structure of the ammonia dimer and the energy difference between the "linear" and "cyclic" configurations. We found that the relative enegetics are well represented at the MP2 level of theory [when compared with higher correlation methods such as CCSD(T)] but the basis set effects are quite dramatic. For instance, basis sets as large as aug-cc-pV5Z are needed in order to accurately reproduce the experimentally obtained energy difference between the two conformations $\left(7.5 \mathrm{~cm}^{-1}\right)$. To this end, the use of the parallel computer and appropriate electronic structure software tailored for the environment (NWCHEM) is a necessity.

The structure of the ammonia trimer is cyclic with a homodromic hydrogen bonding network like the one found in the water trimer (see Figure 3). However, unlike the water trimer that possesses no symmetry elements, $\left(\mathrm{NH}_{3}\right)_{3}$ has $\mathrm{C}_{3}$ symmetry. The contraction of the $\mathrm{N}-\mathrm{N}$ bond lengths from the dimer to the trimer is just $0.065 \AA$, almost half than the one observed for $\left(\mathrm{H}_{2} \mathrm{O}\right)_{3}$ $(0.12 \AA)$. This is an indication that the cooperative effect should be smaller. The electronic

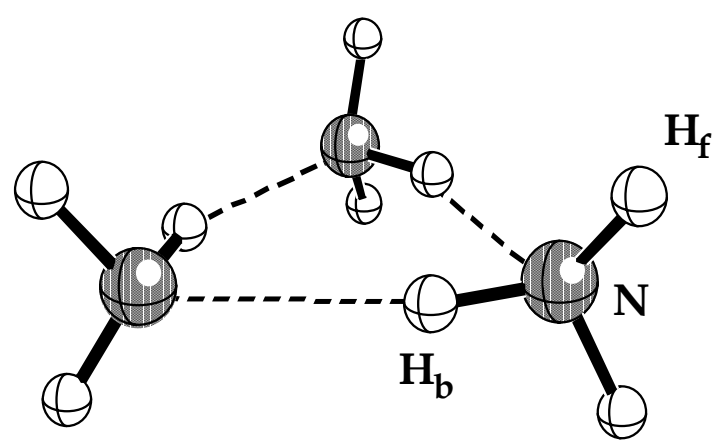

$\mathbf{H}_{\mathbf{f}}$

Figure 3. Equilibrium structure of the ammonia trimer. energy difference with respect to three isolated fragments at the MP2/aug-cc-pVQZ level of theory is $-10.76 \mathrm{kcal} / \mathrm{mol}(-10.56 \mathrm{kcal} / \mathrm{mol}$ including BSSE corrections). Zero-point energy corrections produce $\Delta \mathrm{E}_{0}=-6.8 \mathrm{kcal} / \mathrm{mol}$. Analysis of the nonadditive component of the interaction energy suggests that the three-body term of $-1.19 \mathrm{kcal} / \mathrm{mol}$ is $11 \%$ of the total energy (compared to $17 \%$ for the water trimer). The interaction in $\left(\mathrm{NH}_{3}\right)_{3}$ is smaller than the water trimer as a consequence of the weaker both 2- and 3-body interactions. To this end it appears that cooperative effects manifested in small 
ammonia clusters are not as large as the ones previously observed for water clusters. Future studies of larger ammonia clusters will be used in order to validate this proposition.

For $\mathrm{NH}_{3}-\mathrm{H}_{2} \mathrm{O}$ the electronic energy difference $\left(\Delta \mathrm{E}_{\mathrm{e}}\right)$ was estimated at $-6.5 \mathrm{kcal} / \mathrm{mol}$ at the MP2 compete basis set (CBS) limit (see Figure 4). The optimal structure corresponds to a quasilinear hydrogen bond with the water molecule acting as the proton donor and the ammonia molecule as the proton acceptor. The minimum energy structure on the dimer PES corresponds to a configuration in which the "free" $\mathrm{H}$ atom of the water molecule is in an eclipsed conformation with one of the ammonia $\mathrm{H}$ atoms.

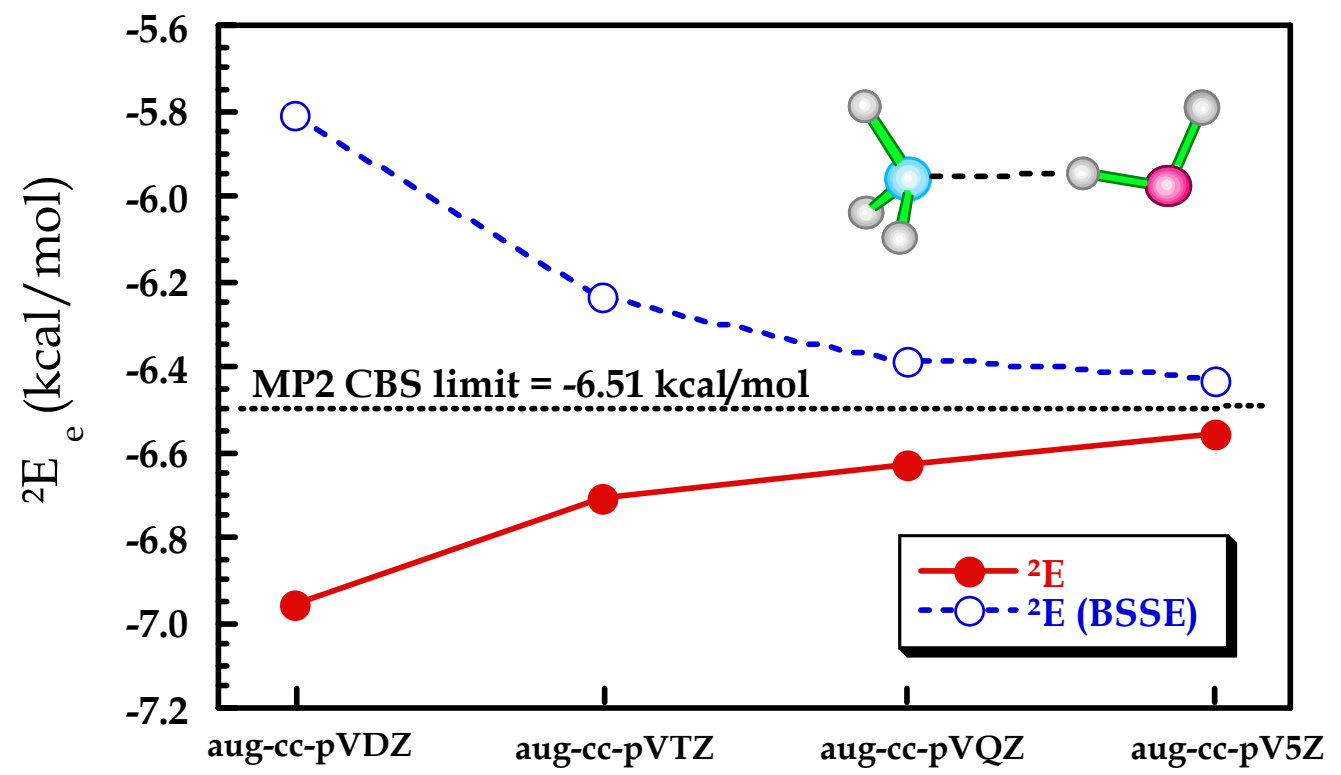

Figure 4. Convergence of ammonia-water binding energy with basis set. The filled circles and solid curve are the MP2 energies, and the open circles and dashed line are the MP2 energies, which are corrected for basis, set superposition error. The dot-dash line is an exponential extrapolation of the MP2 energies to the complete basis set limit.

However the dimer "structure" is by no means rigid since, for instance, rotation of the "free" $\mathrm{H}$ atom of the water molecule along the molecular axis of the ammonia molecule (almost collinear with the $\mathrm{N}-\mathrm{O}$ vector) is quite feasible having an electronic energy barrier of just $0.01 \mathrm{kcal} / \mathrm{mol}$. The dependence of the interaction potential on the change of this dihedral angle is much smaller than the corresponding one in the water dimer. This requires further investigation in order to obtain an orientational interaction potential that correctly reproduces this effect. Zero-point corrections estimated at the harmonic approximation yield $\Delta \mathrm{E}_{0}=-4.3 \mathrm{kcal} / \mathrm{mol}$ for the dimer.

Higher order correlation effects estimated at the CCSD(T) level of theory suggest that the MP2 estimate for $\Delta \mathrm{E}_{\mathrm{e}}$ is accurate to within $0.2 \mathrm{kcal} / \mathrm{mol}$. The interaction is ca. $25 \%$ stronger than the one between two water molecules, a consequence of the fact that ammonia is a better base than water. It should be emphasized that previous estimates for $\Delta \mathrm{E}_{\mathrm{e}}$ of this system (performed at much lower levels of theory than the one used here) range from -4 to $-7.5 \mathrm{kcal} / \mathrm{mol}$ and in many cases they even produce a different qualitative picture for the features of the ground state PES associated with the different conformers. 


\section{Appendix B - Full Report of Second Year Activities and Accomplishments}

\section{$\underline{\text { Nucleation of Tropospheric Aerosols }}$}

We have developed a new theoretical approach to homogeneous gas-to-vapor nucleation that goes well beyond the classical theory of nucleation and also removes approximations of other molecular theories. We adopt the usual starting point for molecular approaches, which is the assumption that addition and removal of monomers from clusters adequately describes the nucleation kinetics. The nucleation rate is obtained by solving the pseudo-first order kinetic equations (assuming the monomer concentration of the nucleating species is large compared to the cluster concentrations and can be approximated as a constant). The first major approximation in other molecular theories is that the condensation rate constant can be approximated by the collision rate of monomers with the cluster. This formulation for the condensation rate constant approximates the cross section for collisions by the surface area of a sphere of radius $\mathrm{r}$, and assumes unit sticking probability upon collision. The evaporation rate constant is then evaluated using detailed balance and the task then reduces to evaluating the equilibrium cluster distribution or equivalently the free energies for forming the clusters from monomers. Because the clusters that are important in the nucleation process can dissociate, the equilibrium cluster distribution or Helmholtz free energy exhibits a dependence on the constraining volume and actually diverges as the volume containing the cluster increases. Treatment of the volume dependence of this free energy has been a source of confusion and controversy in the development of a general molecular theory of nucleation.

In contrast to previous theories, our new molecular theory of nucleation provides a method for directly calculating the evaporation and condensation rate constants. This approach has two major advantages over the previous molecular approaches. First, the direct evaluation of the rate constants avoids approximating the condensation rate constant using a simple gas collision rate. Second, the formulation of the rate constants naturally yields a consistent prescription for dealing with the volume dependence of the free energy for cluster formation and actually exploits this dependence.

We view the processes of evaporation and condensation as gas-phase dissociation and association reactions. This view of the nucleation process as a gas-phase reaction mechanism composed of dissociation and association reactions is quite different than the perspective generally encountered in the nucleation literature where nucleation is viewed as density fluctuations in a supersaturated fluid. Given the large distance between water monomers and clusters in the vapor $\left(>10^{4}-10^{5} \AA\right.$ for water at a supersaturation of 5$)$, the view of nucleation as a gas-phase reaction mechanism is quite reasonable. Transition state theory (TST) provides a convenient framework to evaluate equilibrium rate constants for these kinetic processes.

The TST rate constant is approximated by the equilibrium one-way flux through a dividing surface separating reactants from products. For evaporation of a monomer from an i-molecule cluster, reactants are an i-molecule cluster and products are a monomer infinitely separated from an (i-1)-molecule cluster. In the present work, we choose the dividing surface to be a sphere with its center at the center of mass of the i-molecule cluster; the radius of the sphere then determines the location of the dividing surface. (Spherical dividing surfaces are chosen to make 
contact with previous work that used spherical constraining volumes as well as the simplicity is lends to the theory.) For the dissociation reaction studied here, the location of the dividing surface is guided by variational TST (VTST). The fundamental assumption of TST guarantees that the TST approximation to the classical reactive flux is greater than or equal to the exact classical flux. This upper bound is the basis for variational TST in which the transition state dividing surface is located to minimize the reactive flux. Thus, VTST gives a prescription for defining a unique location of the dividing surface (or radius of the sphere) for each reaction.

In the new theory the rate constant for monomer evaporation from an i-molecule cluster is given by

$$
\alpha_{i}(T)=-\left.\left(\frac{1}{2 \pi m k_{B} T}\right)^{1 / 2} \frac{d}{d r} A_{i}(T, r)\right|_{r=r_{i}^{a ̀ ~}}
$$

where $\mathrm{m}$ is the monomer mass, $\mathrm{k}_{\mathrm{B}}$ is Boltzmann's constant, $\mathrm{T}$ is temperature, and $\mathrm{A}_{\mathrm{i}}(\mathrm{T}, \mathrm{r})$ is the Helmholtz free energy for forming an i-molecule cluster in a sphere of radius $r$. The value of $r$ that minimizes the rate constant is denoted $r_{i}^{a ̀}$. It is worth noting that free energy curves $A_{i}(T, r)$ have been computed by several groups dating back to the early 1970s, but it is only our recent work that has shown the evaporation rate constant can be related to the derivative of $A_{i}(T, r)$ with respect to $\mathrm{r}$. Once the evaporation rate constant is obtained, the condensation rate constant can be obtained (for monomer addition to an (i-1)-cluster) using detailed balance. The resulting expression for the condensation rate requires evaluation of the absolute value of the Helmholtz free energy, whereas the evaporation rate constant requires its derivative with respect to $r$.

The absolute value of the free energy and its derivative are obtained by Monte Carlo simulations. Accurate calculations of $\mathrm{A}_{\mathrm{i}}(\mathrm{T}, \mathrm{r})$ vs. $\mathrm{r}$ require extensive simulations that sample configurations with water molecules evaporated (or with their center of mass at least 4- $6 \AA$ from the nearest neighbor's center of mass) as well as those in which all water molecules are hydrogen bonded together. The configuration space of the i-molecule cluster is sampled in an extended canonical ensemble in which the volume is allowed to fluctuate under imposed constant pressure and constant temperature using Metropolis Monte Carlo. During the course of the Monte Carlo simulation over configurations and volume, the range or volume (or r) is partitioned into bins for accumulation of population. The relative probability of a cluster forming within a given volume $\mathrm{v}$ (or spherical radius $r$ ) is obtained from the normalized population and the relative value of the Helmholtz free energy is obtained from the probability. NWMPP1 provides an excellent platform on which to perform these simulations since the total sampling can be split up over many processors. In order to obtain good statistics many independent simulations are performed and the results from each simulation averaged.

A summary of our results to date for water clusters with 2 to 15 molecules are presented in Table II. Calculation of the equilibrium constants and condensation rate constants requires determining the absolute value of the Helmholtz free energies between adjacent size clusters. To date these calculations have only been carried out through $i=10$. New simulation methods to 
Table II. Summary of the radii $r_{i}^{a}$, Helmholtz free energies $A\left(T, r_{i}^{a}\right)$, VTST evaporation rate constant $\alpha_{\mathrm{i}}$, equilibrium constant $\mathrm{K}_{\mathrm{i}, \mathrm{i}-1}^{\mathrm{EQ}}$, and VTST condensation rate constant $\beta_{\mathrm{i}}$ for i-molecule water clusters at $243 \mathrm{~K}$ and monomer concentration $\rho_{1}=1.5 \times 10^{17} \mathrm{~cm}^{-}$ ${ }^{3}$ using the Dang-Chang model for water interaction energies.

\begin{tabular}{rccclc}
\hline \hline $\mathrm{i}$ & $\begin{array}{c}\mathrm{r}_{\mathrm{i}}^{\mathrm{a}} \\
(\AA)\end{array}$ & $\begin{array}{c}\mathrm{A}\left(\mathrm{T}, \mathrm{r}_{\mathrm{i}}^{\mathrm{a}}\right) \\
(\mathrm{kcal} / \mathrm{mol})\end{array}$ & $\begin{array}{c}\alpha_{\mathrm{i}} \\
\left(10^{9} \mathrm{~s}^{-1}\right)\end{array}$ & $\mathrm{K}^{\mathrm{EQ}_{\mathrm{i}, \mathrm{i}-1}}$ & $\begin{array}{c}\beta_{\mathrm{i}-1} \\
\left(10^{8} \mathrm{~s}^{-1}\right)\end{array}$ \\
\hline 2 & 2.8 & -8.8 & 160 & 0.00041 & 0.68 \\
3 & 4.3 & -17.0 & 49 & 0.0034 & 1.6 \\
4 & 5.8 & -26.5 & 4.7 & 0.038 & 1.8 \\
5 & 5.8 & -35.7 & 9.9 & 0.022 & 2.2 \\
6 & 6.3 & -44.9 & 13 & 0.020 & 2.6 \\
7 & 6.8 & -54.3 & 9.4 & 0.030 & 2.9 \\
8 & 7.5 & -63.9 & 7.2 & 0.036 & 2.6 \\
9 & 8.0 & -73.7 & 5.3 & 0.063 & 3.3 \\
10 & 8.5 & -83.6 & 4.6 & 0.060 & 2.7 \\
11 & 8.5 & & 4.4 & & \\
12 & 9.0 & & 4.2 & & \\
13 & 9.5 & & 3.5 & & \\
14 & 9.5 & & 3.0 & & \\
15 & 9.5 & & 3.3 & & \\
\hline \hline
\end{tabular}

more efficiently calculate absolute free energies are being explored (see below). Calculations have also been performed for the $\mathrm{i}=20$ and 30 clusters yielding $\alpha_{20}=9.2 \times 10^{8} \mathrm{~s}^{-1}$ and $\alpha_{30}=5.4 \times 10^{8} \mathrm{~s}^{-1}$.

We have used the computed rate constants and extrapolated values for larger clusters, to compute the transient rate of water nucleation at $243 \mathrm{~K}$ and monomer concentration $\rho_{1}=1.5 \times 10^{17}$ $\mathrm{cm}^{-3}$. The rate constants and nucleation rates are presented in Figure 5. For the current extrapolation, the critical cluster occurs for about $\mathrm{i}=23$, where the evaporation and condensation rate constants are nearly equal. The cluster populations are seen to rapidly converge to the steady state value of $4 \times 10^{3} \mathrm{~cm}^{-3} \mathrm{~s}^{-1}$ within about $100 \mathrm{ps}$, defining the nucleation rate for these conditions. Given the delicate nature of the nucleation process it is important to assess the effects of uncertainties in the kinetic parameters that govern the steady-state nucleation rate.

Sensitivity analysis provides insight into the influence and identity of the dynamical quantities to which the nucleation rate, $J$, is most sensitive. This, in turn, aids in the elucidation of specific reaction mechanisms and reduces computational effort by focusing only on those clusters that affect the nucleation rate. This analysis revealed two classes of sensitivity: (1) when the nucleation rate is expressed as a function of the evaporation $(\alpha)$ and condensation rate constants $(\beta), J=J(\alpha, \beta)$, then the nucleation rate is most sensitive to all pre-critical clusters, and (2) when the nucleation rate is expressed as a function of the condensation rate constants and equilibrium cluster populations $\left(\mathrm{N}^{\mathrm{EQ}}\right), J=J\left(\beta, \mathrm{N}^{\mathrm{EQ}}\right)$, then the sensitivity is peaked about the critical cluster (see Figure 6). Thus, if the dynamical properties of the clusters around the critical size can be 

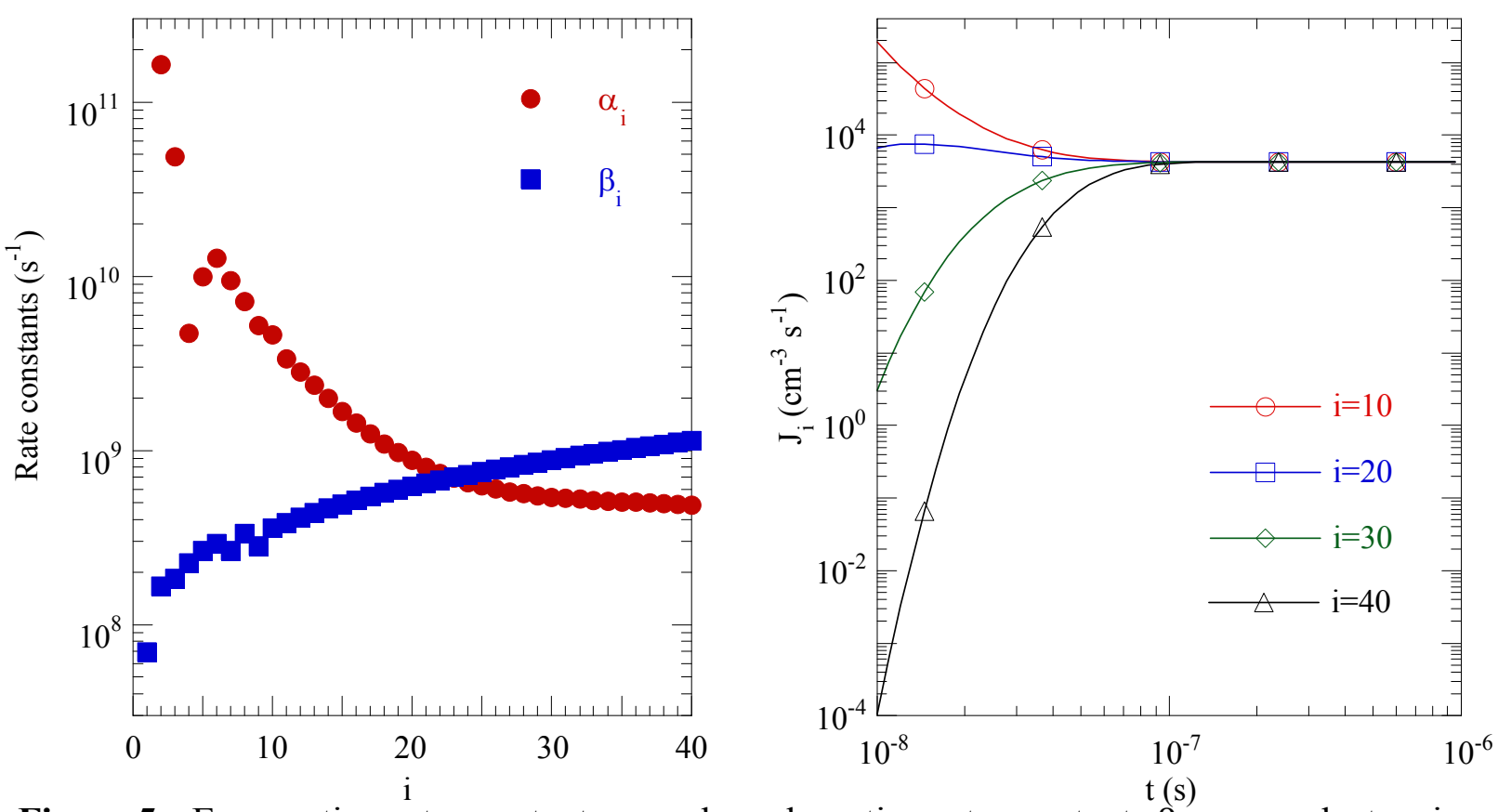

Figure 5. Evaporation rate constants $\alpha_{i}$ and condensation rate constants $\beta_{i}$ versus cluster size (left figure) and transient cluster populations versus time for $\mathrm{i}=10,20,30$, and 40 for water nucleation at $243 \mathrm{~K}$ and monomer concentration of $1.5 \times 10^{17} \mathrm{~cm}^{-3}$.

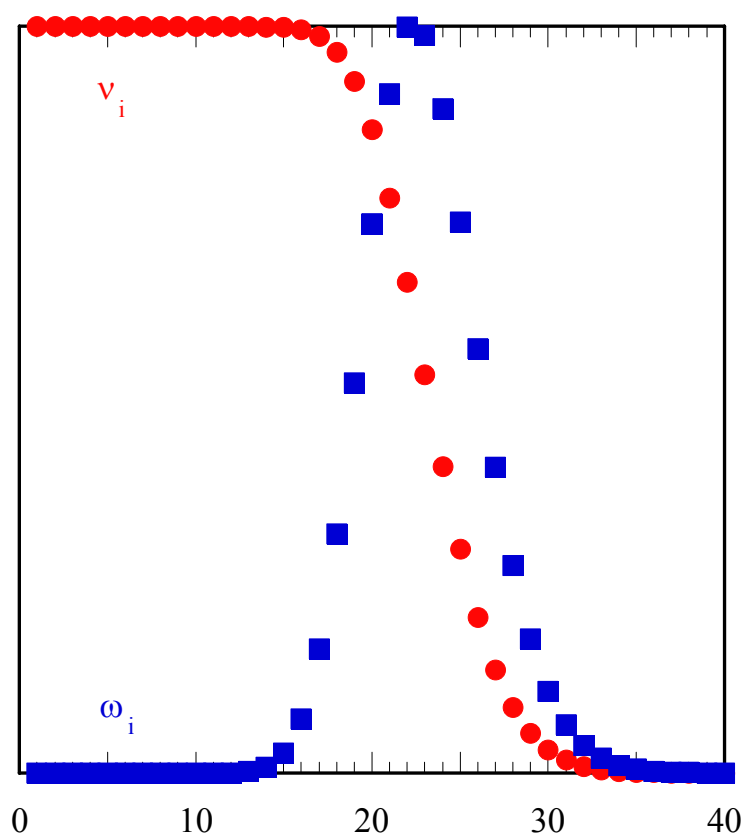

Figure 6. Sensitivity of nucleation with respect to variations of the evaporation and condensation rate constants $\left(v_{i}\right)$ and with respect to variations of the condensation rate constants and equilibrium populations $\mathrm{N}_{\mathrm{I}}^{\mathrm{EQ}}$.

determined directly, then the sensitivity of the nucleation rate on the pre-critical clusters can be avoided. But, this requires the ability to determine the condensation rate constants and populations for clusters containing 30 to 50 water molecules. In addition, the absolute Helmholtz free energies of these clusters must be calculated in order to determine their populations.

The calculation of the water cluster absolute Helmholtz free energy and its radial dependence requires extensive sampling of configuration space. The Northwest Massively Parallel Processor (NWMPP) provides an ideal platform on which to perform Monte Carlo cluster simulations. In order to obtain good statistics many independent trajectories need to be sampled and the results averaged. We have already devised a simulation technique that provides the radial dependence of the Helmholtz free energy; however, we are currently exploring a novel technique that (in principle) can provide the absolute Helmholtz 
free energies. This technique provides an estimate of the absolute Helmholtz free energy via determination of the external work. ${ }^{1}$ In short, since $\Delta \mathrm{E}=\mathrm{Q}+\mathrm{W}$, and $\mathrm{W} \geq \Delta \mathrm{A}$ (where $\Delta \mathrm{E}$ is the change in internal energy of the system, $\mathrm{Q}$ is the heat absorbed by the system, $\mathrm{W}$ is the work done on the system, and $\Delta \mathrm{A}$ is the change in Helmholtz free energy of the system), one can obtain upper and lower bounds for $\Delta \mathrm{A}$ by doing work on the system along a reversible path. That is to say, by slowly changing the underlying potential energy surface from an ideal gas to a fully interacting water cluster an upper bound on $\Delta \mathrm{A}$ can be obtained (the lower bound is obtained by reversing the procedure). By accumulating the total heat (-Q) released to the bath during this process and given $\Delta \mathrm{E}=\mathrm{E}$ (final) $-\mathrm{E}$ (initial), the external work is determined by $\mathrm{W}=\Delta \mathrm{E}+\mathrm{Q}$. A Monte Carlo code was modified to perform external work calculations on water clusters. By using many different starting configurations a thorough sampling of the reversible paths which join the two thermodynamic states of interest can be achieved.

Much effort has been expended towards the development of methods for computing free energy changes via Monte Carlo or Molecular Dynamics simulations. All methods require a choice of a thermodynamic path upon which the free energy is changing from the initial to the final state. Eventhough the free energy is a state function which allows any path connecting the initial and final states as a valid path, there exists the possibility that some particular set of paths may be more computationally efficient. Recall from the earlier discussion that the external work, W, done on a system in raising its free energy from an initial to a final state is, in general, greater than the corresponding change in free energy $\Delta \mathrm{A}$. The work recovered during a decrease in free energy will be less than the free energy change. Thus, one can exploit the fact that energy is lost in the forward and reverse cycles by accumulating the work performed on (or by) the system as an upper (lower) bound on the free energy change: $-\mathrm{W}$ (final $\Rightarrow$ initial) $\leq \Delta \mathrm{A} \leq \mathrm{W}$ (initial $\Rightarrow$ final). Note that the inequalities are saturated only for reversible paths. In other words, the external work is equal to the change in free energy if the system remains at equilibrium along the thermodyanmic path chosen. Hence, one should choose a path which allows the system to remain arbitrarily close to equilibrium while the underlying potential energy surface is altered from the initial to the final state. The way the underlying potential is altered from the initial to final state determines the path (i.e. the external work is a functional of the path). Therefore, the best path is found by changing the way the potential is altered and choosing the case which yields the most rapid convergence of the upper and lower bounds on the free energy difference. It must be stated that it is the ensemble averaged external work which is optimized by path variation. Thus, many initial configurations must be sampled and followed to their final states to achieve a reasonable estimate of the free energy difference. Currently, we are exploring different ways of changing the underlying potential to yield an optimal reversible path. Preliminary results for the Helmholtz free energy difference between the interacting and noninteracting small water clusters at $\mathrm{T}=243 \mathrm{~K}$ are: $\Delta \mathrm{A}($ dimer $)=-1.16 \pm 0.05 \mathrm{kcal} / \mathrm{mol} ; \Delta \mathrm{A}($ trimer $)$ $=-2.26 \pm 0.08 \mathrm{kcal} / \mathrm{mol} ; \Delta \mathrm{A}($ tetramer $)=-5.91 \pm 0.08 \mathrm{kcal} / \mathrm{mol} ; \Delta \mathrm{A}($ pentamer $)=-8.21 \pm 0.05$ $\mathrm{kcal} / \mathrm{mol}$.

As a means of assessing the accuracy of the VTST calculations, we have performed classical trajectory calculations that allow determination of the exact classical rate constants and we have calculated a classical recrossing correction factor $\kappa^{\text {Recross }}$, i.e., $\mathrm{k}^{\mathrm{Cl}}=\kappa^{\text {Recross }} \mathrm{k}^{\text {VTST }}$. Detailed balance insures that the recrossing factors for the evaporation and condensation rate constants are the same. Since evaporation is an infrequent event, we use classical trajectories to simulate the 
condensation reaction. Trajectories are initiated with one water significantly far from the cluster so that the interaction is negligible. The water molecule has its initial geometry and relative translational velocity vector selected in the following manner. A straight line from the center of mass of the cluster is constructed and the initial geometry is chosen to be displaced from this line by the distance $b$, called the impact parameter. The initial relative translational velocity vector is parallel to the line (see Figure 7). For a fixed value of the impact parameter an ensemble of initial conditions of the cluster and incident water molecule are chosen from a Boltzmann distribution and the probability of the water molecule sticking to the cluster is just the number of trajectories for which the incident water molecule sticks divided by the total number of trajectories. Calculated sticking probabilities $\mathrm{P}(\mathrm{b})$ for water collision with $\mathrm{i}=9$ and 29 cluster are shown in Figure 8. For comparison we also indicate the values of $r_{\text {cut }}$ as vertical arrows and the sticking probabilities assumed in classical nucleation theory (unit sticking probability for collision with a water droplet with the density of bulk water). Even for head-on collisions $(b=0)$ the sticking probabilities are not unity. For values of $b$ larger than $r_{\text {cut }}$ a significant fraction of collisions still lead to sticking because of the attractive interaction of the incident water molecule with water molecules near the surface of the cluster. For sufficiently large impact parameter the sticking probability goes to zero as the interaction between the incident water molecule and the cluster is insufficient to attract the incident molecule to the cluster. An accurate classical value of the condensation rate constant $\beta$ is obtained from the sticking probability from the relationship

$$
\beta=\bar{c} \rho_{1} \int_{0}^{\infty} d b 2 \pi b P(b)
$$

where $\overline{\mathrm{c}}$ is the average speed of water molecules in the gas phase and $\rho_{1}$ is the density of water monomers in the gas phase. Table III compares the accurate condensation rate constants with those obtained from VTST and classical nucleation theory (CNT). The VTST results are seen to overestimate the accurate classical results by $60 \%$ for $i=9$ and about a factor of 2 for $i=29$. VTST is seen to get qualitatively correct results for the current choice of dividing surface, but either improved dividing surfaces or classical trajectory calculations of recrossing factors will be needed to get quantitatively accurate rate constants. 


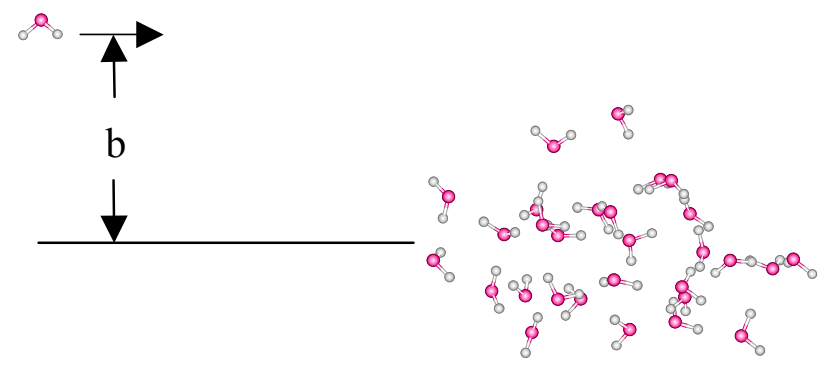

Figure 7. Snapshot of initial geometry for a water molecule collision with a 29water cluster.

Table III. Comparison of condensation rate constants $\beta_{\mathrm{i}}$ for $\mathrm{i}=9$ and 29 calculated from accurate classical trajectories (accurate), variational transition state theory (VTST), and classical nucleation theory (CNT)

\begin{tabular}{|c|c|c|}
\hline \multirow[b]{3}{*}{ Accurate } & \multicolumn{2}{|c|}{$\beta_{\mathrm{i}}\left(10^{8} \mathrm{~s}^{-1}\right)$} \\
\hline & $\mathrm{i}=9$ & $i=29$ \\
\hline & $1.7 \pm 0.3$ & $3.6 \pm 0.7$ \\
\hline VTST & 2.8 & 8.7 \\
\hline CNT & 0.43 & 0.90 \\
\hline
\end{tabular}

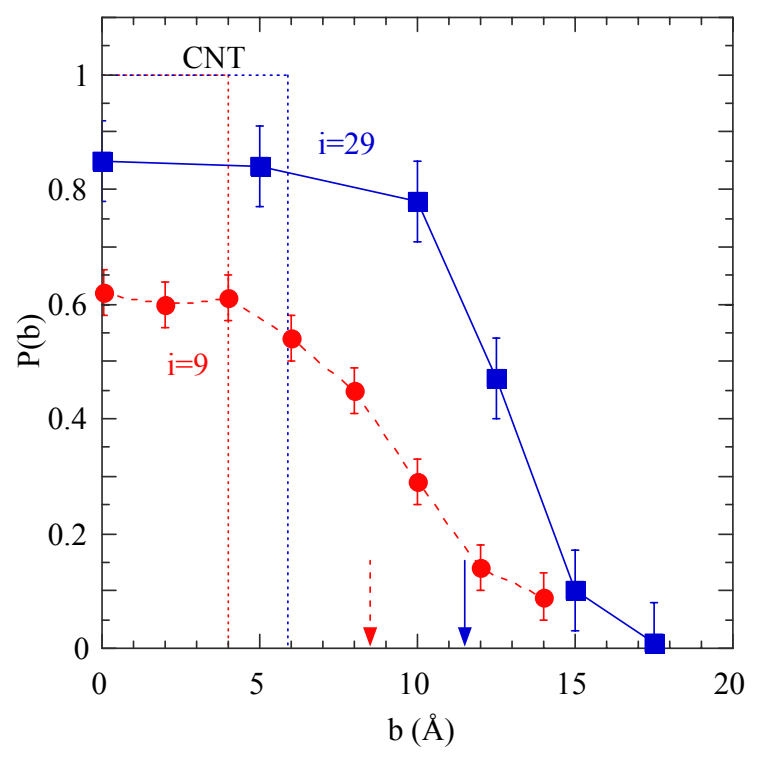

Figure 8. Sticking probability versus impact parameter for water collision with i-molecule water clusters. Circles and squares are classical trajectory results for $\mathrm{i}=9$ and 29 , respectively. The dashed and solid vertical arrows show the values of $r_{\text {cut }}$ for the $i=9$ and 29 clusters. The dashed lines are represent the sticking probabilities implied in classical nucleation theory (with the vertical line at the smaller $b$ value for $\mathrm{i}=9$. 


\section{Appendix C - Full Report of Third Year Activities and Accomplishments}

\section{$\underline{\text { Nucleation of Tropospheric Aerosols }}$}

Considerable effort was made in the third year of this project to implement the finite-time variational switching formalism (also known as the external work technique) ${ }^{1}$ for calculating the absolute value of the Helmholtz free energy and its derivative, which is needed for calculating the evaporation rate constant. To evaluate the success of this approach, we first performed calculations on Lennard-Jones clusters, since an extensive body of knowledge is available for Lennard-Jones fluids and clusters. The Lennard-Jones potential can be used as a simple model of argon interactions, enabling comparison with experiments, but another virtue is its amenability to all types of simulation, providing contact with many different approaches to nucleation theory. The aim of the present work is to produce the first extensive and systematic predictions for argon nucleation from a microscopic theory. Such results should provide a benchmark for comparison with other theories and a basis for refining these theories.

In the external work method the Hamiltonian of the system is gradually changed between the state of interest and a reference state whose free energy is known. The accumulated potential energy changes due to incrementing the Hamiltonian at instantaneously fixed configuration correspond to the irreversible work required to take the system between the two Hamiltonians. The work constitutes an upper bound to the free energy change, and a lower bound can be obtained by switching in the reverse direction, i.e. from the reference state back to the state of interest. A convenient reference state for calculating the absolute free energy of a hot Lennard-Jones cluster is the ideal gas, where the atomic interactions are completely switched off. With a suitable choice of switching path ${ }^{2}$ the free energy difference from the interacting cluster can be calculated efficiently and to arbitrary precision, complete with accurate error estimates. The free energy of the ideal gas constrained within a sphere centered at its center of mass can be evaluated precisely, ${ }^{3}$ allowing the free energy of the Lennard-Jones cluster to be put on an absolute scale. In addition, we calculate the derivative, $\partial \mathrm{A} / \partial \mathrm{R}$, of the Helmholtz free energy with respect to the container radius, by evaluating $A(R)$ over a range then differentiating numerically. The finitetime switching method can also be used in this case by treating the interaction of the atoms with the wall as part of the Hamiltonian. By reducing the radius of the container during the simulation, the walls do work on the cluster, and one obtains the free energy change as a function of radius. The reversibility of this process is greatly enhanced by the newly introduced method of metric scaling. ${ }^{4}$

The finite-time variational method was implemented in parallel on the MPP and the absolute free energies and their derivatives were calculated for argon clusters at $85 \mathrm{~K}$. Condensation and evaporation rate constants were obtained for clusters of 9 to 44 atoms at $85 \mathrm{~K}$. The preliminary results described here indicate the utility of the approach. Future work is aimed at obtaining nucleation rates for argon for a variety of physical conditions (temperature and pressure). In addition, the external work method has been extended to the study of water clusters.

2 W. P. Reinhardt, M. A. Miller and L. M. Amon, Acc. Chem. Res. 34 607-614 (2001)

3 J.. K. Lee, J. A. Barker and F. F. Abraham, J. Chem. Phys. 58, 3166-3180 (1973)

4 M. A. Miller and W. P. Reinhardt, J. Chem. Phys. 113, 7035-7046 (2000) 
A primary focus for the third year of this project was addressing the extreme sensitivity of the nucleation rate to kinetic parameters. Since the kinetic parameters are sensitive to the underlying potential energy surface (PES) of the system, the nucleation rate also exhibits significant sensitivity to the PES. All our previously reported calculations on water clusters have been performed using the Dang-Chang polarizable water model. We tested the sensitivity of the Helmholtz free energies on intermolecular potential by comparing the values for the DangChang potential with those obtained using the TIP4P water model. The Helmholtz free energies for the TIP4P and Dang-Chang water models at a temperature T=243K and corresponding simulation radii $\mathrm{r}_{\text {cut }}$ for clusters of size $i=2$ to 10 are shown in Table IV. These calculations are based on the method of external work and have an error of about $\pm 0.1 \mathrm{kcal} / \mathrm{mol}$. One can readily see that even though these water models give nearly equivalent descriptions of bulk properties, their cluster properties are quite different. However, this situation is not too surprising since neither of these water models used cluster data in the parameterization.

Table IV. Helmholtz free energies (units of $\mathrm{kcal} / \mathrm{mol}$ ) at radii $\mathrm{r}_{\mathrm{i}}^{\grave{a}}$ for $\mathrm{i}$-molecule water clusters at $243 \mathrm{~K}$ using the TIP4P and Dang-Chang water models.

\begin{tabular}{rccc}
\hline \hline $\mathrm{i}$ & $\mathrm{r}_{\mathrm{i}}^{\grave{a}}(\AA)$ & \multicolumn{2}{c}{ Helmholtz free energy } \\
\cline { 3 - 4 } & & TIP4P & $\begin{array}{c}\text { Dang- } \\
\text { Chang }\end{array}$ \\
\hline 2 & 2.8 & -2.2 & -1.2 \\
3 & 4.3 & -5.5 & -2.7 \\
4 & 5.8 & -9.2 & -5.3 \\
5 & 5.8 & -13.0 & -8.3 \\
6 & 6.3 & -16.3 & -11.1 \\
7 & 6.8 & -19.9 & -13.8 \\
8 & 7.5 & -23.4 & -16.1 \\
9 & 8.0 & -26.9 & -19.1 \\
10 & 8.5 & -30.6 & -21.7 \\
\hline \hline
\end{tabular}

Development of an accurate water potential remains a challenge, but once consistency is achieved between nucleation theory and experiment we will have exquisitely nailed down the intermolecular potential for water.

Another major objective of this project is to develop a molecular-level theoretical formulation of aerosol nucleation that demonstrates an understanding of the factors controlling aerosol formation in binary and ternary systems (e.g., systems containing two and three nucleating components). With this objective in mind, we previously extended DNT to multicomponent systems in the past year, and in the third year the multicomponent theory was implemented in the simulation codes. Work has also been proceeding to fit a polarizable ammonia-water potential and a dissociable water-sulfuric acid potential. 


\section{Heterogeneous Reactions of Atmospheric Significance}

In the third year of the project, we have constructed ${ }^{5}$ an explicit time dependent version of the theory of Sum Frequency Generation (SFG) spectroscopy, in order to remove various approximations that we employed in our first order theory published in $2000 .{ }^{6}$ An SFG spectrum provides a surface sensitive probe of the vibrational spectrum of a surface. The removal of these approximations was necessary in order to have a general treatment that should be applicable to complex surfaces, such as sulfate aerosols, and our overall goal is to have a reliable theory to help understand such atmospheric aerosol surfaces and reactions on them.

The new theory still employs quantum chemical electronic structure calculations and molecular dynamics computer simulations, but is framed in a time dependent formulation, which has allowed us to incorporate such important features as electronic polarization of the molecules, intra- and inter-molecular vibrational couplings, and an improved modelling of the vibrational frequency shifts due to these interactions. As an illustration of the new approach, we have focussed on the water surface, and have employed the flexible, anharmonic force field model of water developed by Ferguson, ${ }^{7}$ which is a revised version of the well known SPC model to incorporate intramolecular vibrations, and has been parametrized via neutron diffraction experiments on water. A test calculation of the bulk water infrared spectrum was in good agreement with experiment, and indicated the importance of including the electronic polarization in the calculations. We also examined the technique, introduced by Benjamin in an important first work, ${ }^{8}$ of trying to generate an SFG spectrum by calculation of the ordinary IR spectrum, but restricted to the surface region. We found that no such truncated region IR spectrum could successfully reproduce the calculated SFG spectrum. This indicates that one will require a theory for the SFG spectrum itself, such as the one we have developed.

The comparison of our calculated water surface SFG spectrum with experiment was quite encouraging, although there are discrepancies, to be returned to below. It was found, by comparison with a calculation with a different internal flexible force field (Kuchitsu-Morino ${ }^{9}$ ), not parameterized for the liquid state, that it is quite important to use a force field such as the Ferguson model parametrized for the liquid state. It was also found that inclusion of the electronic polarization is necessary for agreement with experiment. In our comparison with experiment, the new theory worked quite well in the high frequency stretching region of the socalled 'dangling bond' $\mathrm{OH}$, and less well in the so-called 'hydrogen bonding ' region of the spectrum, at lower frequencies. For the former region, we believe that the new theory should be directly applicable, without further development, to more complex surfaces, and studies of these are underway, beginning with a test calculation on the methanol-water surface, which is already

5 A.Morita, J.T. Hynes, A theoretical analysis of the sum frequency generation spectrum of the water surface II. Timedependent approach.

6 A.Morita, J.T. Hynes, A theoretical analysis of the sum frequency generation spectrum of the water surface, Chem. Phys. 258 (2000) 371--390.

7 D.M. Ferguson, Parameterization and evaluation of a flexible water model, J. Comp. Chem. 15 (1995) 501--511.

8 I.Benjamin, Vibrational spectrum of water at the liquid/vapor interface, Phys. Rev. Lett. 73 (1994) 2083-2086.

9 K.Kuchitsu, Y.Morino, Estimation of anharmonic potential constants. II. bend XY2 molecules, Bull. Chem. Soc. Jpn. 38 (1965) 814--824. 
quite well understood from a simulation point of view; application to the sulfate aerosol surface will follow. Concerning the lower frequency 'hydrogen bonded' region, the remaining discrepancies compared to experiment have very few potential sources, given the fact that our new theory has removed almost all approximations. One approximation that does remain is the neglect of induced quadrupole effects (a neglect also made in almost all current qualitative interpretations of SFG spectra). We are currently investigating the impact of this approximation. The potential consequences for the interpretation of SFG are quite significant, in that the induced quadrupole contribution is not restricted to the surface region but also arises from the bulk. If it is found that this contribution is important, then one would have to conclude that, contrary to current thought, SFG is not exclusively a surface sensitive probe for all surfaces in all frequency regions.

Reaction Energetics and Rate Constants for Oxidation of Volatile Organic Compounds Important in the Troposphere

Paulson stated in 1994 that "the ability of a compound to contribute to oxidant formation can only be derived from the detailed oxidation mechanism for each hydrocarbon." For homogeneous gas phase processes these uncertainties lie in the area of degradation mechanisms as opposed to kinetic measurements, although there are still some key kinetic issues that have yet to be resolved. Major areas of mechanistic uncertainty include (1) aromatics, (2) OH chemistry of hydrocarbons $\mathrm{C}_{n}$, with $\mathrm{n}>4$, (3) $\mathrm{O}_{3}$ chemistry of unsaturated hydrocarbons $\mathrm{C}_{\mathrm{n}}$ with $\mathrm{n}>2$, and (4) $\mathrm{OH}, \mathrm{O}_{3}$, and $\mathrm{NO}_{3}$ chemistry of alkenes and terpenes.

During the second year of the project, we initiated exploratory calculations of reaction energetics and rate constants for the reactions of $\mathrm{OH}$ with ethylene, butadiene, isoprene, and $\alpha$-pinene. The major focus of this work has been the exploring theoretical approaches to obtain accurate energetic information for these important reactions. Radical reactions are difficult to study both experimentally and theoretically. Experimentally, radical reactions are very fast, complicating the identification of reaction pathways and intermediates. Theoretically, an accurate description of the low-energy barriers requires sophisticated treatment of electron correlation. The test reaction $\mathrm{OH}+\mathrm{C}_{2} \mathrm{H}_{4}$ was chosen because (1) of its simplicity and (2) there exists experimental rate constants for comparison. Initially, calculations were performed at the MP2//cc-pvdz level that gave a barrier of $7.5 \mathrm{kcal} / \mathrm{mol}$ and a resulting rate constant that was eleven orders of magnitude below experiment. Using the optimized geometries at the MP2 level single point calculations were carried out at the UCCSD(T)//avdz level. The resulting energetics resulted in the barrier being reduced to $-1 \mathrm{kcal} / \mathrm{mol}$ (i.e., below the energy of reactants); however, the rate constant was too slow by an order of magnitude and the temperature dependence was in the wrong direction compared to experiment. Since optimization of the reaction path at the $\mathrm{UCCSD}(\mathrm{T})$ level is intractable, the use of the DFT with the MPW1K functional of Truhlar and coworker was explored. The resulting

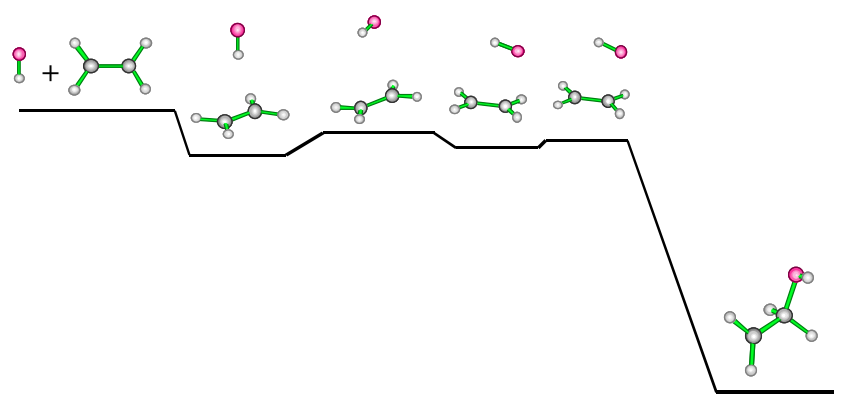

Figure 9. Energy profile for reaction of $\mathrm{OH}+$ ethylene at the DFT/MPW1K level of theory. 
reaction path had the correct negative barrier with respect to reactants by $-1.9 \mathrm{kcal} / \mathrm{mol}$ and the variational transition state theory (VTST) estimate for the rate agrees well with experiment: $k^{\mathrm{VTST}}=6.3 \times 10^{-12} \mathrm{cc} / \mathrm{molecule} / \mathrm{s}$ at $300 \mathrm{~K}$ compared to $k^{\mathrm{EXP}}=\left(4.5 \times 10^{-12}\right.$ to $\left.9.9 \times 10^{-12}\right)$ $\mathrm{cc} / \mathrm{molecule} / \mathrm{s}$. This reaction goes through a van der Waals complex $(-3.2 \mathrm{kcal} / \mathrm{mol})$, through a first transition state $(-1.9 \mathrm{kcal} / \mathrm{mol})$, into a $2^{\text {nd }}$ complex $(-2.2 \mathrm{kcal} / \mathrm{mole})$, through a $2^{\text {nd }}$ transition state $\left(\sim 5 \times 10^{-3} \mathrm{kcal} /\right.$ mole above the $2^{\text {nd }}$ complex $)$, and then to the product $\mathrm{C}_{2} \mathrm{H}_{4} \mathrm{OH}(-33.6$ $\mathrm{kcal} / \mathrm{mol}$ ) as shown schematically in Figure 9. Furthermore, the theoretical rate constants are consistent with the measured temperature dependence. These results represent the most comprehensive study of $\mathrm{OH}+\mathrm{C}_{2} \mathrm{H}_{4}$ reaction energetics to date. A previous investigation of this reaction by J. Villa et al. (JCP, 107 (18), 7266, (1997)) considered only $0.5 \AA$ along the reaction path and thus completely missed both the $2^{\text {nd }}$ complex and $2^{\text {nd }}$ transition state. Calculations are underway to benchmark these latest results against those at the UCCSD(T) level of theory. In addition, calculations for the stationary points along the reaction path for $\mathrm{OH}+$ butadiene are currently being performed. 